\title{
Gravitational shockwaves on rotating black holes
}

\author{
Yoni BenTov* \\ Institute for Quantum Information and Matter, \\ California Institute of Technology, Pasadena, CA 91125 \\ Joe Swearngin \\ Department of Physics, \\ University of California, Santa Barbara, CA 93106
}

\begin{abstract}
We present an exact solution of Einstein's equation that describes the gravitational shockwave of a massless particle on the horizon of a Kerr-Newman black hole. The backreacted metric is of the generalized Kerr-Schild form and is Type II in the Petrov classification. We show that if the background frame is aligned with shear-free null geodesics, and if the background Ricci tensor satisfies a simple condition, then all nonlinearities in the perturbation will drop out of the curvature scalars. We make heavy use of the method of spin coefficients (the Newman-Penrose formalism) in its compacted form (the Geroch-Held-Penrose formalism).
\end{abstract}

\section{Contents}

\section{Contents}

1 Motivation

2 The Kerr-Newman black hole 5

2.1 Null frame . . . . . . . . . . . . . . . . . . . . 5

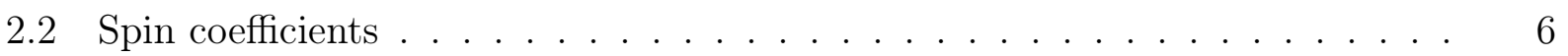

2.3 Partial gauge fixing . . . . . . . . . . . . . . . . . . . . 7

2.4 Matter fields and gauge fields . . . . . . . . . . . . . . . . 9 9

2.5 Null Cartan equations . . . . . . . . . . . . . . . . . . . 9 9

2.6 Timelike expansion and timelike twist . . . . . . . . . . . . . . 10

2.7 Kruskal-like coordinates . . . . . . . . . . . . . . . . 11

2.8 A smooth frame . . . . . . . . . . . . . . . . . . . . 12

2.9 Spacelike and timelike curvatures . . . . . . . . . . . . . 13

${ }^{*}$ Current affiliation: Perimeter Institute for Theoretical Physics, Waterloo, ON N2L 2Y5, Canada 
2.10 Curvature scalars . . . . . . . . . . . . . . . . . 14

2.11 Gravitational compass and Petrov classification . . . . . . . . . . 15

2.12 Energy scalars . . . . . . . . . . . . . . . . . 16

3 Shifted frame and Kerr-Schild form 16

3.1 From Reissner-Nordström to Kerr-Newman . . . . . . . . . . . . . . . . . . . 17

3.2 Preliminary commentary . . . . . . . . . . . . . . . . . 18

3.3 Shifted spin coefficients . . . . . . . . . . . . . . . . . 18

3.4 Shifted horizon ......................... 21

3.5 Refraction ........................ 21

4 Petrov classification for the Kerr-Newman shockwave 21

4.1 Shifted $\Psi_{4}$ and physical interpretation . . . . . . . . . . . . 21

4.2 Nonrotating limit . . . . . . . . . . . . . . . . . . 23

4.3 Shifted $\Psi_{3}$ and Petrov type . . . . . . . . . . . . . . . . 23

4.4 Curvatures of submanifolds . . . . . . . . . . . . . . . . . 23

4.5 Shifted $\Psi_{2} \ldots \ldots \ldots \ldots \ldots$

5 Shifted Ricci scalars 24

5.1 Ricci scalar of weight $(-1,-1)$ : Absence of nonlinearity . . . . . . . . . 24

5.2 Other Ricci scalars . . . . . . . . . . . . . . . . 25

6 Derivatives of the shift $\quad 26$

6.1 Key facts . . . . . . . . . . . . . . . . . . . 27

6.2 Integration by parts . . . . . . . . . . . . . . . . 27

6.3 First-derivative terms . . . . . . . . . . . . . . . . . . 28

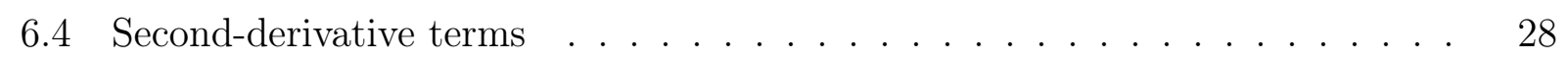

6.5 Transverse box . . . . . . . . . . . . . . . . . . . . . . . 29

6.6 Laplacian on the squashed sphere . . . . . . . . . . . . 30

7 Ricci tensor $\quad 30$

7.1 Relation to curvature scalars . . . . . . . . . . . . . . . . . . . 30

7.2 Relation to energy scalars ......................... 31

7.3 Final result for $\Phi_{22} \ldots \ldots \ldots \ldots \ldots$. . . . . . . . . . . . . . . . . . . . . .

8 Discussion $\quad 34$

A Signature change

A.1 Basic assumptions . . . . . . . . . . . . . . . . . . . . . . . . . . . . . . . . . .

A.2 Spin coefficients flip sign .................... 35

A.3 Curvature scalars do not flip sign . . . . . . . . . . . . . . . 36

A.4 Extra sign in GHP derivatives . . . . . . . . . . . . . . 36

References 


\section{Motivation}

Black holes are thermodynamic systems whose microscopic description we still do not understand. After the original work on black hole thermodynamics by Christodoulou [1], Penrose and Floyd [2], Carter [3], Bekenstein [4], and Bardeen, Carter, and Hawking [5], Hawking justified the analogy between the surface gravity $\left.\right|^{1} \alpha$ and a temperature $T$ by predicting that an isolated black hole will radiate as a black body at the expected temperature $T=\frac{\alpha}{2 \pi}[6,7]$. About 20 years later, Strominger and Vafa vindicated the analogy between the horizon area $A$ and an entropy $S$ by enumerating microstates in string theory to derive the expected result $S=\frac{1}{4} A$ for extremal black holes in $4+1$ dimensions 8.

We will not recount the subsequent history of microstate counting. Suffice it to say that the calculations from string theory, while eminently laudable, are restricted to black holes near extremality and may not provide enough insight into the statistical mechanics behind the conventional black holes of general relativity for generic values of their parameters. It would be helpful to establish a complementary strategy for black hole statistical mechanics tailored to an expansion around the Schwarzschild solution.

One such alternative is the S-matrix approach of 't Hooft $[9,10]$. Motivated by this and by Shenker and Stanford's investigation of the butterfly effect [11, 12], Kitaev recently proposed a quantum field theory in $0+1$ dimensions [13] whose low-energy effective action is that of dilaton gravity in $1+1$ dimensions 14,15 . Details of this model were explored further by Maldacena and Stanford [16]. Since the equations of motion derived from the effective action admit the $\mathrm{AdS}_{2}$ black hole as a solution [17], Kitaev's calculation demonstrates that the thermodynamic limit of a quantum mechanical mode $\mathrm{I}^{2}$ can produce a bona fide black hole horizon, albeit in lower-dimensional scalar-tensor gravity, not in $(3+1)$-dimensional Einstein gravity.

Foundational to all of this is an exact solution of Einstein's equation that describes the gravitational backreaction of a massless particle on the future horizon of a Schwarzschild black hole: the Dray-'t Hooft gravitational shockwave [19] $]^{3}$

That solution was generalized to the Reissner-Nordström (RN) black hole by Alonso and Zamorano [22] and by Sfetsos [23], who also adapted the shockwave to other static backgrounds. Kiem, Verlinde, and Verlinde 24] used a perturbative variant of the Dray-'t Hooft result to see how gravitational interactions might affect black hole evaporation. And Polchin-

\footnotetext{
${ }^{1}$ We use " $\alpha$ " instead of the more conventional " $\kappa$ " for surface gravity because " $\kappa$ " has been commandeered by Newman and Penrose (see Sec. 2.4).

${ }^{2}$ As remarked by Witten, "the average of a quantum system over quenched disorder is not really a quantum system" 18. Strictly speaking it is only a quantum mechanical model if the average captures the physics of a single realization with fixed couplings $J_{j k \ell m}$. We thank Yonah Lemonik for a discussion about this important point.

${ }^{3}$ This solution can be viewed as the generalization of the Aichelburg-Sexl shockwave [20] to curved spacetime or as an application of Penrose's "scissors-and-paste" method for gluing together known solutions of Einstein's equation to form new solutions 21.
} 
ski 25] revisited the solution to refine 't Hooft's "relation between a given black hole S-matrix element and another with an additional ingoing particle," culminating in a reformulated argument for the firewall 26,27 .

In his exposition of the S-matrix framework, 't Hooft did not concern himself with more general black hole backgrounds, opining that "[c]onceptually, generalization of everything we say to these cases should be straightforward" [10]. Perhaps, but in this paper our principal ambition is to galvanize the search for a statistical mechanics underlying astrophysical black holes [28], whose equilibrium field configurations are described by the Kerr geometry. So if we intend to adapt 't Hooft's blueprint and Kitaev's recent insights to the microscopics of rotating black holes, then our very first preliminary step must be to generalize 't Hooft's formula for the transition amplitude.

That is what we do here: We generalize the Dray-'t Hooft gravitational shockwave to the Kerr-Newman background, which is the most general asymptotically flat black hole in four spacetime dimensions. Readers familiar with gravitational shockwaves and the method of spin coefficients could skip to our metric ansatz described by Eqs. (3.1) and (3.7), and then to our main result: the Ricci tensor in Eq. (7.3), the Ricci scalar $\Phi_{22}$ in Eq. (7.11), and the differential operator in Eq. (7.12). We acknowledge that this provides only the most tentative intimation toward a microscopic theory of the Kerr-Newman spacetime, but it is a new exact solution of Einstein's equation and therefore deserves to be studied in its own right.

Only late in our venture did we learn that Balasin generalized the Ricci tensor for the Dray't Hooft solution with the express aim of including rotation in the formalism $[29]$. But he did not complete the calculation, stating only that "it would be interesting to apply it to a rotating, i.e. Kerr black hole" and that "[w]ork in this direction is currently in progress." Similar comments were made by Alonso and Zamorano [22] and by Taub [30]. We have not found later articles by any of these authors that contain our results.

In Sec. 2, we review everything required to follow the calculation - those unfamiliar with null frames will likely have to supplement this with standard references like Chandrasekhar [31] and Penrose and Rindler [32]. In Sec. 3 we recast the Dray-'t Hooft geometry as a shift of the null frame, explain how to include rotation, and compute the spin coefficients for generalized Kerr-Schild metrics. In Secs. 4 and 5 we specialize to shear-free geodesic congruences and compute the shifted curvature scalars. Sec. 6 is where the heavy lifting begins: We engage the rotating shockwave and compute some preliminary identities for derivatives of the shift function. This leads to Sec. 7), where we complete the calculation and announce the differential equation for the shockwave's angular profile. We offer some closing thoughts in Sec. 8, and we explain in the appendix how to change metric signature from mostly-minus to mostly-plus.

\footnotetext{
${ }^{4}$ We found Balasin's paper after we had already computed the Ricci tensor but before we managed to express it in the relatively compact and geometrical form described by Eqs. $7.37,(7.11)$, and 7.12 .
} 


\section{The Kerr-Newman black hole}

To enable the reader to work through this document, we will first describe the Kerr-Newman black hole using the method of spin coefficients.

This method was invented by Newman and Penrose (NP) [33] and refined into a "compacted" version by Geroch, Held, and Penrose (GHP) [34], a refinement that has since fallen by the wayside but that we found indispensable. Beside our primary aim of generalizing the gravitational shockwave, our secondary aim is to provide a detailed example of how to use the formalism. As far as rotating black holes are concerned, the flip side of the method of spin coefficients is the madness without it.

\section{$2.1 \quad$ Null frame}

Our account of the spacetime will begin with a collection of frame field 1-forms

$$
e^{a} \equiv e_{\mu}^{a} d x^{\mu} \equiv\left(-l^{\prime},-l, m^{\prime}, m\right)
$$

in terms of which the line element is

$$
d s^{2}=-2 l l^{\prime}+2 m m^{\prime}
$$

A tactical advantage of deploying a frame formulation is to never have to look at a line element, so we will not show $d s^{2}$ explicitly — we will always work directly with the frame. To gain our footing we will start with the "Schwarzschild-like" coordinates $(t, r, \theta, \varphi)$ of Boyer and Lindquist [35], which are applicable outside the black hole.

Kerr-Newman black holes have a mass $M$, a charge $Q$, and an angular momentum $J$. It is customary to trade $J$ for the ratio $a \equiv J / M$ and to define the "horizon function" 31 |

$$
\Delta \equiv r^{2}-2 M r+a^{2}+Q^{2} \equiv\left(r-r_{+}\right)\left(r-r_{-}\right)
$$

The inner horizon $r_{-} \equiv M-\sqrt{M^{2}-a^{2}-Q^{2}}$ and the outer horizon $r_{+} \equiv M+\sqrt{M^{2}-a^{2}-Q^{2}}$ are defined as the solutions to $\Delta=0$. It is useful to note that $M=\frac{1}{2}\left(r_{+}+r_{-}\right)$and $\|(a, Q)\| \equiv\left(a^{2}+Q^{2}\right)^{1 / 2}=\left(r_{+} r_{-}\right)^{1 / 2}$.

We will be concerned exclusively with the region $r \geq r_{+}$, so when we refer to "the" horizon, we will always mean the outer one.

Since time immemorial Newman has emphasized that rotating black holes are "complex translations" of nonrotating ones [36]. Regardless of whether that means anything, it is convenient to define the complex functions

$$
R \equiv r+i a \cos \theta, \quad R_{0} \equiv r+i a .
$$


In the above notation, the following null 1-forms describe the Kerr-Newman black hole:

$$
\begin{aligned}
& l=-d t+\frac{|R|^{2}}{\Delta} d r+a \sin ^{2} \theta d \varphi, \quad l^{\prime}=\frac{\Delta}{2|R|^{2}}\left(-d t-\frac{|R|^{2}}{\Delta} d r+a \sin ^{2} \theta d \varphi\right) \\
& m=\frac{1}{R \sqrt{2}}\left(|R|^{2} d \theta+i\left|R_{0}\right|^{2} \sin \theta d \varphi-i a \sin \theta d t\right), m^{\prime}=m^{*} .
\end{aligned}
$$

Given those 1-forms, we solve the matrix inversion problem

$$
e_{\mu}^{a} e_{a}^{\nu} \equiv \delta_{\mu}^{\nu}, \quad e_{a}^{\mu} e_{\mu}^{b} \equiv \delta_{a}^{b}
$$

for the vectors $e_{a}^{\mu} \equiv\left(l^{\mu}, l^{\prime \mu}, m^{\mu}, m^{\prime \mu}\right)$. By royal mandate we then introduce the NewmanPenrose directional derivatives:

$$
D \equiv l^{\mu} \nabla_{\mu}, \quad D^{\prime} \equiv l^{\prime \mu} \nabla_{\mu}, \quad \delta \equiv m^{\mu} \nabla_{\mu}, \quad \delta^{\prime} \equiv m^{\prime \mu} \nabla_{\mu}
$$

Without loss of generality we can replace the covariant derivatives by partial derivatives and treat the operators $D, D^{\prime}, \delta, \delta^{\prime}$ as ordinary vector fields..$^{5}$ In Schwarzschild-like coordinates, we have:

$$
\begin{aligned}
& D=l^{\mu} \partial_{\mu}=\frac{\left|R_{0}\right|^{2}}{\Delta} \partial_{t}+\partial_{r}+\frac{a}{\Delta} \partial_{\varphi}, \quad D^{\prime}=l^{\prime \mu} \partial_{\mu}=\frac{\Delta}{2|R|^{2}}\left(\frac{\left|R_{0}\right|^{2}}{\Delta} \partial_{t}-\partial_{r}+\frac{a}{\Delta} \partial_{\varphi}\right), \\
& \delta=m^{\mu} \partial_{\mu}=\frac{1}{R \sqrt{2}}\left(\partial_{\theta}+\frac{i}{\sin \theta} \partial_{\varphi}+i a \sin \theta \partial_{t}\right), \delta^{\prime}=\delta^{*}
\end{aligned}
$$

We will refer to the forms in Eq. (2.5) and the vectors in Eq. (2.8) as the "standard" frame. Its ubiquity derives from its utility: It is a principal basis (see Sec. 2.11) whose outgoing and ingoing null congruences are geodesic, twisting, and shear-free [see Eq. (2.33)]. Students acquainted with Reissner-Nordström but hesitant about Kerr-Newman should fiddle with the standard frame until the geometry feels less foreign.

\subsection{Spin coefficients}

There are two ways to express the classical field theory of gravity, distinguished by whether local invariance under $S O(3,1)$ is imposed or inferred. Drastically oversimplifying a complicated history, we will say that the former is Cartan's approach, while the latter is Einstein's.6

We favor the former. First introduce a frame $e_{\mu}^{a}$ and demand invariance of the action under local $S O(3,1)$ transformations:

$$
e^{a}(x) \rightarrow O_{b}^{a}(x) e^{b}(x), \quad O_{c}^{a}(x) O_{d}^{b}(x) \eta_{a b} \equiv \eta_{c d} .
$$

\footnotetext{
${ }^{5}$ Once the equations of differential geometry are cast in spin coefficient form, all of the dynamical variables will be invariant under coordinate transformations on the base space, thereby becoming scalar fields.

${ }^{6}$ For example, Penrose and Rindler 32 refer to what we call "Cartan's approach" as the "Einstein-CartanSciama-Kibble theory" (see their Sec. 4.7).
} 
Then introduce an $S O(3,1)$ gauge field $\omega_{b}^{a}$, called the spin connection, to turn ordinary derivatives into covariant derivatives. As for any nonabelian gauge field, the required transformation law is

$$
\omega^{a}{ }_{b}(x) \rightarrow O^{a}{ }_{c}(x)\left(\delta^{c}{ }_{d} d+\omega^{c}{ }_{d}\right)\left(O^{-1}\right)_{b}^{d}(x) .
$$

By birthright the spin connection is antisymmetric:

$$
\omega_{a b}=-\omega_{b a} .
$$

The variables $e^{a}(x)$ and $\omega_{b}^{a}(x)$ are the independent classical fields in the action. Because we find it productive to work entirely within the tangent space, we follow Newman and Penrose and define the spin coefficients 33.

$$
\gamma_{a b c} \equiv\left(\omega_{\mu}\right)_{a b} e_{c}^{\mu}
$$

Varying the action with respect to the spin connection in a world without fermions implies the torsion-free condition

$$
d e_{a}=\gamma_{a b c} e^{b} \wedge e^{c} .
$$

Solving this gives the spin coefficients in terms of the frame:

$$
\gamma_{a b c}=\frac{1}{2}\left(\lambda_{a b c}+\lambda_{c a b}-\lambda_{b c a}\right), \quad \lambda_{a b c} \equiv-\left(e_{a}^{\mu} e_{c}^{\nu}-e_{c}^{\mu} e_{a}^{\nu}\right) \partial_{\mu} e_{b \nu} .
$$

While this expression is standard, the path to it depends on one's taste in formalism.

\subsection{Partial gauge fixing}

After Newman and Penrose invented the method of spin coefficients, Geroch, Held, and Penrose recognized that specifying a frame $e_{a}^{\mu}=\left(l^{\mu}, l^{\prime \mu}, m^{\mu}, m^{\prime \mu}\right)$ that satisfies the normalization conditions in Eq. 2.6) only partially fixes the gauge in $S O(3,1)$.

The remaining ambiguity comprises a boost along the outgoing congruence, the corresponding inverse boost along the ingoing congruence, and a rotation of the transverse plane:

$$
l^{\mu} \rightarrow r(x) l^{\mu}, \quad l^{\prime \mu} \rightarrow \frac{1}{r(x)} l^{\prime \mu}, m^{\mu} \rightarrow e^{i \vartheta(x)} m^{\mu}, m^{\prime \mu} \rightarrow e^{-i \vartheta(x)} m^{\prime \mu}
$$

We will say that this transformation generates the GHP group. It is convenient to define the complex function

$$
\lambda \equiv r^{1 / 2} e^{i \vartheta / 2}
$$

and to rewrite Eq. (2.15) as

$$
l^{\mu} \rightarrow \lambda \lambda^{*} l^{\mu}, l^{\prime \mu} \rightarrow \lambda^{-1} \lambda^{*-1} l^{\prime \mu}, m^{\mu} \rightarrow \lambda \lambda^{*-1} m^{\mu}, m^{\prime \mu} \rightarrow \lambda^{-1} \lambda^{*} m^{\prime \mu} .
$$

We will say that a function $f_{h, \bar{h}}$ transforms as the representation $]^{7}(h, \bar{h})$ of the GHP group if its transformation law under Eq. (2.17) has the form:

$$
f_{h, \bar{h}} \rightarrow \lambda^{2 h} \lambda^{* 2 \bar{h}} f_{h, \bar{h}}
$$

\footnotetext{
${ }^{7}$ The bar is part of the name of the weight and does not denote any sort of conjugation.
} 
As shorthand for this, we will use the standard notation of representation theory:

$$
f_{h, \bar{h}} \sim(h, \bar{h})
$$

The numbers $(h, \bar{h})$ are called the weight: 8 of the function $f_{h, \bar{h}}$, and such a function is accordingly said to be "weighted." Borrowing group-theoretic jargon from field theory, we will say that weighted quantities transform as matter fields. An object that cannot be assigned a transformation law of the form in Eq. (2.18) for any values of $(h, \bar{h})$ will be called "nonweighted.' 9 In the language of Eq. (2.19), we summarize Eq. 2.17) as

$$
l^{\mu} \sim\left(\frac{1}{2}, \frac{1}{2}\right), l^{\prime \mu} \sim\left(-\frac{1}{2},-\frac{1}{2}\right), m^{\mu} \sim\left(\frac{1}{2},-\frac{1}{2}\right), m^{\prime \mu} \sim\left(-\frac{1}{2}, \frac{1}{2}\right) .
$$

Manifest covariance under the GHP group is what defines the compacted formalism: All explicitly written quantities transform according to Eq. (2.18) for some values of $h$ and $\bar{h}$. Only objects with the same weights can be added, and the weights of a product of objects are the sums of the weights of each object:

$$
f_{h_{1}, \bar{h}_{1}} \sim\left(h_{1}, \bar{h}_{1}\right), g_{h_{2}, \bar{h}_{2}} \sim\left(h_{2}, \bar{h}_{2}\right) \Longrightarrow f_{h_{1}, \bar{h}_{1}} g_{h_{2}, \bar{h}_{2}} \sim\left(h_{1}+h_{2}, \bar{h}_{1}+\bar{h}_{2}\right) .
$$

From Eq. 2.18) we deduce that complex conjugation exchanges the weights:

$$
f_{h, \bar{h}} \sim(h, \bar{h}) \Longrightarrow\left(f_{h, \bar{h}}\right)^{*} \sim(\bar{h}, h) .
$$

Beside complex conjugation, there are two discrete transformations under which the compacted formalism is covariant. The first is the priming transformation, which is defined to exchange primed and unprimed quantities:

$$
l_{\mu} \leftrightarrow l_{\mu}^{\prime}, m_{\mu} \leftrightarrow m_{\mu}^{\prime}
$$

In this way the notation from Eq. (2.1) becomes an operation. From Eq. (2.17) we deduce that priming flips the signs of the weights:

$$
f_{h, \bar{h}} \sim(h, \bar{h}) \Longrightarrow\left(f_{h, \bar{h}}\right)^{\prime} \sim(-h,-\bar{h}) .
$$

The second discrete transformation is the Sachs operation, which is an analog of Hodge duality:

$$
\left(l_{\mu}, l_{\mu}^{\prime}, m_{\mu}, m_{\mu}^{\prime}\right) \rightarrow\left(m_{\mu},-m_{\mu}^{\prime},-l_{\mu}, l_{\mu}^{\prime}\right) .
$$

Unlike priming, the Sachs operation does not commute with complex conjugation. It is extremely convenient to streamline the spin coefficient formalism by using a notation that is manifestly covariant under priming. The Sachs operation will instead help us establish geometrical meaning.

\footnotetext{
${ }^{8}$ Penrose and Rindler define $p \equiv 2 h$ and $q \equiv 2 \bar{h}$. Either way, the "boost weight" and the "spin weight" are defined as $\frac{1}{2}(p+q)=h+\bar{h}$ and $\frac{1}{2}(p-q)=h-\bar{h}$ respectively 34 .

${ }^{9}$ Something invariant under Eq. 2.18) is considered to be weighted with weight zero, not nonweighted.
} 


\subsection{Matter fields and gauge fields}

Based on their behavior under Eq. (2.17), the 12 independent $\gamma_{a b c}$ fall naturally into three sets: weighted quantities associated with $l_{\mu}$, weighted quantities associated with $l_{\mu}^{\prime}$, and nonweighted quantities that transform as gauge fields.

The weighted spin coefficients associated with $l_{\mu}$, along with their weights, are

$$
\kappa \equiv \gamma_{311} \sim\left(\frac{3}{2}, \frac{1}{2}\right), \tau \equiv \gamma_{312} \sim\left(\frac{1}{2},-\frac{1}{2}\right), \sigma \equiv \gamma_{313} \sim\left(\frac{3}{2},-\frac{1}{2}\right), \rho \equiv \gamma_{314} \sim\left(\frac{1}{2}, \frac{1}{2}\right) .
$$

The weighted spin coefficients associated with $l_{\mu}^{\prime}$ are defined by priming, which flips the signs of the weights:10

$$
\kappa^{\prime} \equiv \gamma_{422} \sim\left(-\frac{3}{2},-\frac{1}{2}\right), \tau^{\prime} \equiv \gamma_{421} \sim\left(-\frac{1}{2}, \frac{1}{2}\right), \sigma^{\prime} \equiv \gamma_{424} \sim\left(-\frac{3}{2}, \frac{1}{2}\right), \rho^{\prime} \equiv \gamma_{423} \sim\left(-\frac{1}{2},-\frac{1}{2}\right) .
$$

The gauge fields of the spin coefficient formalism are defined as

$$
\varepsilon \equiv \frac{1}{2}\left(-\gamma_{121}+\gamma_{341}\right), \beta \equiv \frac{1}{2}\left(-\gamma_{123}+\gamma_{343}\right), \varepsilon^{\prime} \equiv \frac{1}{2}\left(-\gamma_{212}+\gamma_{432}\right), \beta^{\prime} \equiv \frac{1}{2}\left(-\gamma_{214}+\gamma_{434}\right)
$$

These are gauge fields in the sense that they combine with the NP derivatives of Eq. (2.8) to form weighted derivatives:

$$
\begin{aligned}
& \mathrm{p} \equiv D+2 h \varepsilon+2 \bar{h} \varepsilon^{*}, \quad \text { } \equiv \delta+2 h \beta-2 \bar{h} \beta^{*}, \\
& \mathrm{p}^{\prime} \equiv D^{\prime}-2 h \varepsilon^{\prime}-2 \bar{h} \varepsilon^{\prime *}, \gamma^{\prime} \equiv \delta^{\prime}-2 h \beta^{\prime}+2 \bar{h} \beta^{*} .
\end{aligned}
$$

We will refer to the operators $\mathrm{p}, \mathrm{p}^{\prime}, \partial$, and $\chi^{\prime}$ as GHP-covariant derivatives. Typically the covariant derivative of a matter field transforms as the same representation as the field itself, but not so here. For a weighted function $f_{h, \bar{h}} \sim(h, \bar{h})$, we have:

$\mathrm{p} f_{h, \bar{h}} \sim\left(h+\frac{1}{2}, \bar{h}+\frac{1}{2}\right), \quad \mathrm{p}^{\prime} f_{h, \bar{h}} \sim\left(h-\frac{1}{2}, \bar{h}-\frac{1}{2}\right), \quad \partial f_{h, \bar{h}} \sim\left(h+\frac{1}{2}, \bar{h}-\frac{1}{2}\right), \quad \chi^{\prime} f_{h, \bar{h}} \sim\left(h-\frac{1}{2}, \bar{h}+\frac{1}{2}\right)$.

Evidently the covariant derivatives themselves carry charge:

$$
\mathrm{p} \sim\left(\frac{1}{2}, \frac{1}{2}\right), \mathrm{p}^{\prime} \sim\left(-\frac{1}{2},-\frac{1}{2}\right), \quad \text { ठ }\left(\frac{1}{2},-\frac{1}{2}\right), \chi^{\prime} \sim\left(-\frac{1}{2}, \frac{1}{2}\right) .
$$

\subsection{Null Cartan equations}

Expressed in the NP hieroglyphs of Eqs. (2.26)-(2.28), the torsion-free condition of Eq. (2.13) becomes four fundamental relations:

$$
\begin{aligned}
& d l=-2 \operatorname{Re}(\varepsilon) l \wedge l^{\prime}+2 i \operatorname{Im}(\rho) m \wedge m^{\prime}+\left[\left(\tau-\beta+\beta^{* *}\right) m^{\prime} \wedge l+\kappa m^{\prime} \wedge l^{\prime}+c . c .\right], \\
& d m=\left(\beta+\beta^{\prime *}\right) m \wedge m^{\prime}-\left(\tau-\tau^{\prime *}\right) l \wedge l^{\prime}+\left[(\rho-2 i \operatorname{Im}(\varepsilon)) m \wedge l^{\prime}+\sigma m^{\prime} \wedge l^{\prime}+c . c .^{\prime}\right]
\end{aligned}
$$

and their primes. We will call these the null Cartan equations.

\footnotetext{
${ }^{10}$ Priming acts on the tangent-space indices by exchanging $1 \leftrightarrow 2$ and $3 \leftrightarrow 4$. Complex conjugation leaves 1 and 2 fixed while exchanging $3 \leftrightarrow 4$.
} 
By computing the exterior derivatives of the forms in Eq. (2.5), arranging them to match the right-hand sides in Eq. 2.32), and solving the resulting equations, we can find the KerrNewman spin coefficients:

$$
\begin{aligned}
& \kappa=\kappa^{\prime}=\sigma=\sigma^{\prime}=0, \quad \rho=\frac{1}{R^{*}}, \quad \rho^{\prime}=-\frac{\Delta}{2|R|^{2}} \frac{1}{R^{*}}, \quad \tau=\frac{i a \sin \theta}{\sqrt{2}|R|^{2}}, \tau^{\prime}=\frac{i a \sin \theta}{\sqrt{2\left(R^{*}\right)^{2}}}, \\
& \varepsilon=0, \quad \varepsilon^{\prime}=\rho^{\prime}+\frac{2 r-r_{+}-r_{-}}{4|R|^{2}}, \quad \beta=-\frac{\cot \theta}{2 \sqrt{2} R}, \quad \beta^{\prime}=\frac{1}{2} \tau^{\prime}-\frac{r \cos \theta-i a}{2 \sqrt{2}\left(R^{*}\right)^{2} \sin \theta} .
\end{aligned}
$$

Because of their obvious noncovariance under Eq. (2.17), the above $\varepsilon^{\prime}$ and $\beta^{\prime}$ should be understood strictly numerically. Also note that $|\tau|^{2}=\left|\tau^{\prime}\right|^{2}$, which will be useful later.

\subsection{Timelike expansion and timelike twist}

Every bard recounts legends of refraction $(\kappa)$, expansion $(\operatorname{Re} \rho)$, twist $(\operatorname{Im} \rho)$, and shear $(\sigma)$, but nary a soul tells tales of $\tau[1$

We would like to elevate the standing of $\tau$ and $\tau^{\prime}$ to match the renown of their colleagues, because these neglected spin coefficients convey the relativistic effects of rotating bodies at least as directly as $\operatorname{Im}(\rho)$ and $\operatorname{Im}\left(\rho^{\prime}\right)$ do - a cursory assessment of Eq. (2.33), for instance, reveals the suggestive factor $a \sin \theta$. Our North Star will be the Sachs operation of Eq. 2.25.

The combinations $\tau \pm \tau^{*}$, rather than $\tau$ and $\tau^{\prime}$ separately, will appear front and center in the subsequent analysis, so let us consider their meaning and christen them with appropriate names. Sachs conjugation of the expansion and twist provides:

$$
\begin{aligned}
& \operatorname{Re}(\rho) \equiv \frac{1}{2}\left(\rho+\rho^{*}\right) \rightarrow \frac{1}{2}\left(\tau+\tau^{\prime *}\right)=-\frac{a^{2} \sin (2 \theta)}{2 \sqrt{2}|R|^{2} R} \\
& \operatorname{Im}(\rho) \equiv \frac{1}{2 i}\left(\rho-\rho^{*}\right) \rightarrow \frac{1}{2 i}\left(\tau-\tau^{*}\right)=\frac{r a \sin \theta}{\sqrt{2}|R|^{2} R}
\end{aligned}
$$

Consequently, we will refer to $\tau+\tau^{\prime *}$ and $\tau-\tau^{\prime *}$ as the timelike expansion and timelike twist.

Even though we performed the Sachs operation on spin coefficients associated with $l^{\mu}$, the result involved both $\tau$ and $\tau^{\prime}$. While this may be jarring at first sight, GHP covariance requires it: The spin coefficients $\rho$ and $\rho^{*}$ have the same weights and therefore can be added and subtracted at will, but $\tau$ and $\tau^{*}$ transform differently under Eq. (2.17). Only $\tau$ and $\tau^{*}$ can be added and subtracted.

\footnotetext{
${ }^{11}$ Sachs, who pioneered the optical analogy for the spin coefficients, does not explain $\tau$ or $\tau^{\prime}$ in his original paper [37]. Szekeres, in the paper from which we extracted the term "refraction" for $\kappa$, calls the spin coefficient $\tau$ (which he denotes $\Omega$ ) the "angular velocity or rotation of the null congruence," but he does not explain why [38. In a subsequent lecture, Sachs seems to have implicitly recognized this interpretation of $\tau$ by also choosing the symbol $\Omega$ to denote it, but he does not justify the notation [39]. An appraisal of the null Cartan equations within the formal context of lightcone kinematics as originally articulated by Dirac [40] affirms this interpretation but with $\tau$ and $\tau^{\prime}$ switched.
} 


\subsection{Kruskal-like coordinates}

To put all this formalism to work, we will need to forge Kruskal-like coordinates. First recall the known result for the surface gravity:

$$
\alpha=\frac{r_{+}-r_{-}}{2\left(r_{+}^{2}+a^{2}\right)}
$$

With that we define the null coordinates $U$ and $V$ outside the black hole:

$$
U \equiv-e^{-\alpha u}, V \equiv+e^{+\alpha v}, u \equiv t-r_{*}, v \equiv t+r_{*}, \quad d r_{*} \equiv \frac{\left|R_{0}\right|^{2}}{\Delta} d r
$$

Note that $U<0$, which is the standard convention. We choose the integration constant in the tortoise coordinate $r_{*}$ such that the product of $U$ and $V$ is:

$$
U V=-\frac{\Delta}{r_{+} r_{-}}\left(\frac{r}{r_{-}}-1\right)^{-k} e^{2 \alpha r}, \quad k \equiv \frac{r_{-}^{2}+a^{2}}{r_{+}^{2}+a^{2}}+1 .
$$

Considered an implicitly defined function of $U$ and $V$, the coordinate $r$ retains its desirable property from the nonrotating case of depending only on the product $U V$. As written in Eq. 2.37, the ratio $\frac{\Delta}{U V}$ is manifestly finite and nonzero at $r=r_{+}$:

$$
c \equiv-\left.\frac{\Delta}{U V}\right|_{r=r_{+}}=r_{+} r_{-}\left(\frac{r_{+}}{r_{-}}-1\right)^{k} e^{-2 \alpha r_{+}} .
$$

For later convenience, we also differentiate both sides of Eq. (2.37) and rearrange to solve for the partial derivatives of $r(U, V)$ :

$$
U \partial_{U} r=V \partial_{V} r=\frac{\Delta}{\Delta^{\prime}(r)+\left(2 \alpha-\frac{k}{r-r_{-}}\right) \Delta} .
$$

For any function $F(r)$ that depends only on the radial coordinate, we therefore have:

$$
U \partial_{U} F(r)=V \partial_{V} F(r)=F^{\prime}(r) U \partial_{U} r \quad \text { and }\left.\quad U \partial_{U} r\right|_{r=r_{+}}=0
$$

We will sometimes use a subscript "+" to label quantities evaluated at the horizon. For instance, $\left|R_{+}\right|^{2} \equiv r_{+}^{2}+a^{2} \cos ^{2} \theta$ and $\left|R_{0+}\right|^{2} \equiv r_{+}^{2}+a^{2}$.

Finally, we define the delayed angular coordinate and the angular velocity at the horizon:

$$
\chi \equiv \varphi-\Omega_{H} t, \quad \Omega_{H}=\frac{a}{r_{+}^{2}+a^{2}} .
$$

\footnotetext{
${ }^{12}$ Since we always work with $r>r_{-}$, we have dropped the absolute values that emerge from integrating $d r_{*}$. Our coordinates are singular at the inner horizon, and a different set of Kruskal-like coordinates must be established to cross it.
} 


\subsection{A smooth frame}

Smooth coordinates are not enough — we also need a smooth frame. From the standard basis written in Kruskal-like coordinates, we perform the following GHP transformation:

$$
l_{\mu} \rightarrow \hat{l}_{\mu}=-U l_{\mu}, \quad l_{\mu}^{\prime} \rightarrow \hat{l}_{\mu}^{\prime}=-U^{-1} l_{\mu}^{\prime}, \quad m_{\mu} \rightarrow \hat{m}_{\mu}=m_{\mu} .
$$

This describes the special case

$$
\lambda=\lambda^{*}=(-U)^{1 / 2}
$$

of the transformation in Eq. 2.17). A hatted function with weights $(h, \bar{h})$ is then related to its unhatted counterpart by

$$
\hat{f}_{h, \bar{h}}=(-U)^{h+\bar{h}} f_{h, \bar{h}} .
$$

The spin coefficients $\rho$ and $\rho^{\prime}$ in the hatted basis,

$$
\hat{\rho}=\frac{1}{R^{*}}(-U) \text { and } \quad \hat{\rho}^{\prime}=\frac{1}{2|R|^{2}}\left(\frac{\Delta}{U V}\right) \frac{1}{R^{*}} V,
$$

go to zero at the future horizon $(U=0)$ and the past horizon $(V=0)$ respectively. These furnish local definitions for each part of the horizon.

Because $\tau \sim\left(\frac{1}{2},-\frac{1}{2}\right)$ and $\tau^{\prime} \sim\left(-\frac{1}{2}, \frac{1}{2}\right)$, those two spin coefficients are invariant under the rescaling in Eq. (2.42):

$$
\hat{\tau}=\tau, \quad \hat{\tau}^{\prime}=\tau^{\prime} .
$$

After changing coordinates from $(t, r, \theta, \varphi)$ to $(U, V, \theta, \chi)$ and applying Eq. 2.42), we obtain the following frame field 1-forms:

$$
\begin{aligned}
& \hat{l}=\frac{-1}{2 \alpha}\left(1+\frac{|R|^{2}}{\left|R_{0}\right|^{2}}-\Omega_{H} a \sin ^{2} \theta\right) d U-\frac{U}{V}\left(1-\frac{\left|R_{0+}\right|^{2}}{\left|R_{0}\right|^{2}}\right) \frac{a^{2} \sin ^{2} \theta}{2 \alpha\left|R_{0+}\right|^{2}} d V-U a \sin ^{2} \theta d \chi, \\
& \hat{l}^{\prime}=\frac{\Delta}{2|R|^{2}}\left[\frac{1}{2 \alpha}\left(1+\frac{|R|^{2}}{\left|R_{0}\right|^{2}}-\Omega_{H} a \sin ^{2} \theta\right) \frac{d V}{U V}+\frac{1}{U^{2}}\left(1-\frac{\left|R_{0+}\right|^{2}}{\left|R_{0}\right|^{2}}\right) \frac{a^{2} \sin ^{2} \theta}{2 \alpha\left|R_{0+}\right|^{2}} d U-\frac{a \sin ^{2} \theta}{U} d \chi\right], \\
& \hat{m}=\frac{1}{R \sqrt{2}}\left[|R|^{2} d \theta+i\left|R_{0}\right|^{2} \sin \theta d \chi+\frac{i a \sin \theta}{2 \alpha\left|R_{0+}\right|^{2}} \frac{r+r_{+}}{r-r_{-}} \frac{\Delta}{U V}(U d V-V d U)\right] .
\end{aligned}
$$

The corresponding directional derivatives are

$$
\begin{aligned}
& \hat{D}=-2 \alpha\left|R_{0}\right|^{2} \frac{U V}{\Delta} \partial_{V}-a \frac{U}{\Delta}\left(1-\frac{\left|R_{0}\right|^{2}}{a} \Omega_{H}\right) \partial_{\chi}, \\
& \hat{D}^{\prime}=\frac{\Delta}{2|R|^{2}}\left[2 \alpha \frac{\left|R_{0}\right|^{2}}{\Delta} \partial_{U}-\frac{a}{U \Delta}\left(1-\frac{\left|R_{0}\right|^{2}}{a} \Omega_{H}\right) \partial_{\chi}\right], \\
& \hat{\delta}=\frac{1}{R \sqrt{2}}\left[\partial_{\theta}+\frac{i}{\sin \theta} \frac{\left|R_{+}\right|^{2}}{\left|R_{0+}\right|^{2}} \partial_{\chi}+i \alpha a \sin \theta\left(-U \partial_{U}+V \partial_{V}\right)\right] .
\end{aligned}
$$

We will refer to the forms in Eq. (2.47) and the vectors in Eq. (2.48) as the "horizon" frame (or simply as the "hatted" one). Each component of the 1-forms in Eq. (2.47) and of the vectors in Eq. (2.48) is finite at $U=0$ for fixed $V$, and at $V=0$ for fixed $U$. 


\subsection{Spacelike and timelike curvatures}

Commutators of covariant derivatives beget curvature. By composing GHP derivatives on a test function $\xi_{h} \sim(h, 0)$, we define the spacelike and timelike curvatures $\mathcal{K}$ and $\mathcal{K}_{s}$ :

$$
\begin{aligned}
\mathcal{K} \xi_{h} & \equiv-\frac{1}{2 h}\left(\left[\partial, \partial^{\prime}\right]+2 i \operatorname{Im}(\rho) \mathrm{p}^{\prime}-2 i \operatorname{Im}\left(\rho^{\prime}\right) \mathrm{p}\right) \xi_{h}, \\
\mathcal{K}_{s} \xi_{h} & \equiv \frac{1}{2 h}\left(\left[\mathrm{p}, \mathrm{p}^{\prime}\right]+\left(\tau-\tau^{\prime *}\right) \jmath^{\prime}+\left(\tau^{*}-\tau^{\prime}\right) ð\right) \xi_{h} .
\end{aligned}
$$

Twice the real part of $\mathcal{K}$ is the ordinary notion of intrinsic (or "Gaussian") curvature in Riemannian geometry. The imaginary part is an extrinsic quantity that we will call the extrinsic curvature ${ }^{13}$ For Kerr-Newman, the intrinsic and extrinsic curvatures are

$$
\begin{aligned}
\operatorname{Re}(\mathcal{K})=\frac{1}{2|R|^{6}} & \left\{r^{2}\left(r^{2}+a^{2}\right)+\left[\left(r_{+}^{2}+a^{2}\right)-4\left(r_{+} r+a^{2}\right)-\left(r^{2}-r_{+}^{2}\right)\right] a^{2} \cos ^{2} \theta\right. \\
& \left.+4 \alpha\left(r_{+}^{2}+a^{2}\right)\left(r-r_{+}\right) a^{2} \cos ^{2} \theta\right\}
\end{aligned}
$$

and

$$
\begin{aligned}
\operatorname{Im}(\mathcal{K})=\frac{a \cos \theta}{|R|^{6}} & \left\{\left(r-r_{+}\right) r_{+} r+\left(2 a^{2}+r^{2}\right) r-r_{+} a^{2} \cos ^{2} \theta\right. \\
& \left.+\alpha\left(r_{+}^{2}+a^{2}\right)\left[\left(2 r_{+}-r\right) r+a^{2} \cos ^{2} \theta\right]\right\}
\end{aligned}
$$

At the horizon, the intrinsic curvature is 41

$$
\left.\operatorname{Re}(\mathcal{K})\right|_{r=r_{+}}=\frac{\left|R_{0+}\right|^{2}}{2\left|R_{+}\right|^{6}}\left(r_{+}^{2}-3 a^{2} \cos ^{2} \theta\right) .
$$

Only at $r=r_{+}$should the denominations "intrinsic" and "extrinsic" be taken literally, because only there do $m^{\mu}$ and $m^{\prime \mu}$ form a surface. In contrast, the real and imaginary parts of $\mathcal{K}_{s}$ can never be interpreted this way, because $l^{\mu}$ and $l^{\prime \mu}$ never form a surface. ${ }^{14}$ So we leave the Kerr-Newman timelike curvature as a complex quantity:

$$
\begin{aligned}
\mathcal{K}_{s} & =\frac{-2\left(3 r_{+}-2 r\right) r r_{+}+2 i a r_{+}\left(4 r-r_{+}\right) \cos \theta+a^{2}\left[5 r-2 r_{+}-\left(2 r_{+}-r\right) \cos (2 \theta)\right]+2 i a^{3} \cos ^{3} \theta}{4 R^{*}|R|^{4}} \\
& +\alpha\left(r_{+}^{2}+a^{2}\right) \frac{\left(3 r_{+}-r\right) r-i a\left(2 r-r_{+}\right) \cos \theta+a^{2} \cos ^{2} \theta}{R^{*}|R|^{4}}
\end{aligned}
$$

Next we will summarize those remaining aspects of curvature that are pertinent but more or less standard.

\footnotetext{
${ }^{13}$ This is not to be conflated with what numerical relativists call the extrinsic curvature, which is part of the spin connection. See, for example, the discussion of contorted surfaces on p. 400 of Spinors and Spacetime 32 .

${ }^{14}$ Take the hatted basis and consider the commutators of covariant derivatives on a test function of weight $(0,0)$ : We have $\left[\hat{\partial}, \hat{\partial}^{\prime}\right]=\left(\hat{\rho}-\hat{\rho}^{*}\right) \hat{\mathrm{p}}^{\prime}-\left(\hat{\rho}^{\prime}-\hat{\rho}^{\prime *}\right) \hat{\mathrm{b}}$ and $\left[\hat{\mathrm{p}}, \hat{\mathrm{p}}^{\prime}\right]=\left(\hat{\tau}-\hat{\tau}^{\prime *}\right) \hat{\partial}^{\prime}+\left(\hat{\tau}^{*}-\hat{\tau}^{\prime}\right) \hat{\partial}$. The righthand side of the former vanishes at $U=V=0$, while the right-hand side of the latter never vanishes except at the poles. We thank Leo Stein for emphasizing this to us.
} 


\subsection{Curvature scalars}

The Riemann tensor in the tangent space is

$$
R_{a b c d}=\partial_{c} \gamma_{a b d}-\partial_{d} \gamma_{a b c}-\gamma_{a b}^{e}\left(\gamma_{c e d}-\gamma_{d e c}\right)+\gamma_{a e c} \gamma_{b d}^{e}-\gamma_{a e d} \gamma_{b c}^{e}
$$

From the corresponding Ricci tensor, $R_{a b} \equiv R_{a c b}^{c}$, Newman and Penrose define a traceless matrix

$$
\phi_{a b} \equiv \frac{1}{2}\left(R_{a b}-\frac{1}{4} \eta_{a b} \eta^{c d} R_{c d}\right) .
$$

For spinorial reasons of no concern to us, they then define the Ricci scalars as

$$
\begin{aligned}
& \Phi_{00} \equiv \phi_{11} \sim(1,1), \quad \Phi_{01} \equiv \phi_{13} \sim(1,0), \quad \Phi_{02} \equiv \phi_{33} \sim(1,-1) \\
& \Phi_{22} \equiv \Phi_{00}^{\prime}=\phi_{22} \sim(-1,-1), \Phi_{21} \equiv \Phi_{01}^{\prime}=\phi_{24} \sim(-1,0) \\
& \Phi_{20} \equiv \Phi_{02}^{\prime}=\phi_{44}=\Phi_{02}^{*} \sim(-1,1), \Phi_{10} \equiv \Phi_{01}^{*}=\phi_{14} \sim(0,1) \\
& \Phi_{12} \equiv \Phi_{21}^{*}=\phi_{23} \sim(0,-1), \quad \Phi_{11} \equiv \frac{1}{2}\left(\phi_{12}+\phi_{34}\right) \sim(0,0)
\end{aligned}
$$

In the notation of the compacted formalism, we have

$$
\begin{aligned}
& \Phi_{00}=-\mathrm{p} \rho-\rho^{2}-|\sigma|^{2}+\jmath^{\prime} \kappa+\tau^{\prime} \kappa+\tau \kappa^{*}, \Phi_{02}=-ð \tau-\tau^{2}-\kappa \kappa^{\prime *}+\mathrm{p}^{\prime} \sigma+\rho^{\prime} \sigma+\rho \sigma^{* *}, \\
& \Phi_{01}=\frac{1}{2}\left[-\mathrm{p} \tau+\mathrm{p}^{\prime} \kappa-ð \rho+\partial^{\prime} \sigma-\left(\tau-\tau^{*}\right) \rho-\left(\tau^{*}-\tau^{\prime}\right) \sigma-\left(\rho-\rho^{*}\right) \tau+\left(\rho^{\prime}-\rho^{\prime *}\right) \kappa\right] .
\end{aligned}
$$

The remaining Ricci scalars of nonzero weight can be defined by priming and conjugating the definitions already listed: $\Phi_{22}=\Phi_{00}^{\prime}, \Phi_{21}=\Phi_{01}^{\prime}, \Phi_{10}=\Phi_{01}^{*}, \Phi_{12}=\Phi_{21}^{*}$, and $\Phi_{20}=\Phi_{02}^{*}$. Meanwhile, the Ricci scalar of weight $(0,0)$ is defined in terms of the spacelike and timelike curvatures:

$$
\Phi_{11}=\frac{1}{2}\left(\mathcal{K}-\mathcal{K}_{s}-\kappa \kappa^{\prime}+\tau \tau^{\prime}-\sigma \sigma^{\prime}+\rho \rho^{\prime}\right) .
$$

Tradition compels a fanciful notation for a factor times the trace of the Ricci tensor:

$$
\Pi \equiv \frac{1}{12}\left(R_{12}-R_{34}\right)=-\frac{1}{24} \eta^{a b} R_{a b}
$$

Because of its role as the gravitational Lagrangian, we refer to this as the Einstein-Hilbert curvature. In GHP notation, it reads

$$
\Pi=\frac{1}{6}\left[2\left(\rho \rho^{\prime *}-|\tau|^{2}+\mathrm{p}^{\prime} \rho-{\chi^{\prime} \tau}^{\prime}\right)+\mathcal{K}+\mathcal{K}_{s}-\kappa \kappa^{\prime}-\tau \tau^{\prime}+\sigma \sigma^{\prime}+\rho \rho^{\prime}\right] .
$$

Finally we are left with the completely traceless part of the curvature:

$$
C_{a b c d} \equiv R_{a b c d}+\eta_{a d} \phi_{b c}+\eta_{b c} \phi_{a d}-\eta_{a c} \phi_{b d}-\eta_{b d} \phi_{a c}+2\left(\eta_{a c} \eta_{b d}-\eta_{a d} \eta_{b c}\right) \Pi \text {. }
$$

This is the Weyl tensor in the tangent space, and from it Newman and Penrose define the Weyl scalars:

$$
\begin{aligned}
& \Psi_{0} \equiv C_{1313} \sim(2,0), \quad \Psi_{1} \equiv C_{1312} \sim(1,0), \quad \Psi_{2} \equiv C_{1342} \sim(0,0) \\
& \Psi_{3} \equiv \Psi_{1}^{\prime}=C_{2421} \sim(-1,0), \quad \Psi_{4} \equiv \Psi_{0}^{\prime}=C_{2424} \sim(-2,0) .
\end{aligned}
$$

\footnotetext{
${ }^{15}$ The expression for $\Phi_{00}$ is in fact real but not manifestly so.
} 
In GHP notation, the first three of these are $e^{16}$

$$
\begin{aligned}
& \Psi_{0}=-\left[\mathrm{p}+\left(\rho+\rho^{*}\right)\right] \sigma+\left[\partial+\left(\tau+\tau^{\prime *}\right)\right] \kappa, \\
& \Psi_{1}=\frac{1}{2}\left\{-\mathrm{p} \tau+\mathrm{p}^{\prime} \kappa+ð \rho-\chi^{\prime} \sigma-\left(\tau-\tau^{*}\right) \rho-\left(\tau^{*}-\tau^{\prime}\right) \sigma+\left(\rho-\rho^{*}\right) \tau-\left(\rho^{\prime}-\rho^{*}\right) \kappa\right\}, \\
& \Psi_{2}=\frac{1}{3}\left[\rho \rho^{*}-|\tau|^{2}+\mathrm{p}^{\prime} \rho-\partial^{\prime} \tau-\left(\mathcal{K}+\mathcal{K}_{s}\right)-2 \kappa \kappa^{\prime}+\tau \tau^{\prime}+2 \sigma \sigma^{\prime}-\rho \rho^{\prime}\right] .
\end{aligned}
$$

The remaining two are defined by priming.

\subsection{Gravitational compass and Petrov classification}

Szekeres conjured an elegant theoretical apparatus called the gravitational compass to interpret the Weyl scalars [42]. Following his insight, we will say that $\Psi_{2}$ describes a Coulomb field, $\Psi_{4}$ describes a transverse outgoing wave, and $\Psi_{3}$ describes a longitudinal outgoing wave. The primed quantities, $\Psi_{0} \equiv \Psi_{4}^{\prime}$ and $\Psi_{1} \equiv \Psi_{3}^{\prime}$, describe the corresponding ingoing waves ${ }^{17}$

The Weyl scalars are not gauge invariant: A local $S O(3,1)$ transformation $e^{a} \rightarrow O^{a}{ }_{b} e^{b}$ results in $\Psi_{\alpha} \rightarrow \sum_{\beta=0}^{4} Q_{\alpha \beta} \Psi_{\beta}$ for some matrix $Q_{\alpha \beta}$. We can ask how many $\Psi_{\alpha}$ can be simultaneously gauged away, and we can classify spacetimes based on the answer. This is Chandrasekhar's 31] account of the Petrov classification [43] of solutions to the vacuum Einstein equation. A desire to elucidate the physics behind each Petrov type is what drove Szekeres to engineer the gravitational compass.

We will only study two Petrov types: Type D, in which all of the Weyl scalars beside $\Psi_{2}$ can be gauged away, and Type II, in which all of the Weyl scalars beside $\Psi_{2}$ and $\Psi_{4}$ can be gauged away. ${ }^{18}$ Extending the standard terminology slightly beyond its ordinary usage, we will define a principal frame as any basis in which as many Weyl scalars as possible for a given geometry are gauged away.

The Kerr-Newman black hole is Type D, and its nonzero Weyl scalar is

$$
\Psi_{2}=-\frac{1}{\left(R^{*}\right)^{3}}\left(M-\frac{Q^{2}}{R}\right) .
$$

Because it carries charge, this black hole is not a vacuum solution - the Weyl scalars are no longer the whole story. Local sources of energy induce Ricci curvature, and in this case the electromagnetic field induces

$$
\Phi_{11}=\frac{Q^{2}}{2|R|^{4}}
$$

\footnotetext{
${ }^{16}$ Having defined $\Psi_{1}$ and $\Phi_{01}$, we can compose $\mathrm{p}$ and $ð$ on an arbitrarily-weighted test function and deduce the mixed commutator relation

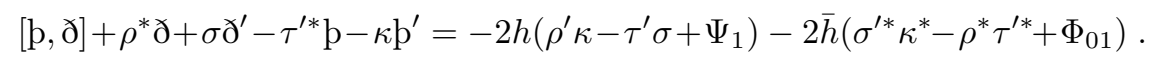

${ }^{17}$ The Coulomb component is self-prime. We might also suggest an alternative notation to make Szekeres's interpretation manifest: $\Psi_{\perp} \equiv \Psi_{4}, \Psi_{\|} \equiv \Psi_{3}, \Psi_{C} \equiv \Psi_{2}, \Psi_{\perp}^{\prime} \equiv \Psi_{0}$, and $\Psi_{\|}^{\prime} \equiv \Psi_{1}$.

${ }^{18}$ For a Type II spacetime, we can rotate the frame to trade a nonzero $\Psi_{4}$ for a nonzero $\Psi_{3}$. This resolves the superficial discrepancy between Chandrasekhar's [31] and Penrose and Rindler's descriptions [32]. Szekeres [42 and Griffiths [44] use the terminology of Penrose and Rindler.
} 


\subsection{Energy scalars}

In the relativistic zeitgeist, the Ricci scalars are considered a stand-in for the energy tensor by means of Einstein's equation. But we find this confusing and will briefly suggest a refined presentation.

To match Penrose's traceless Ricci tensor from Eq. 2.55), we define a traceless energy tensor

$$
\mathcal{T}_{a b} \equiv \frac{1}{2}\left(T_{a b}-\frac{1}{4} \eta_{a b} \eta^{c d} T_{c d}\right)
$$

From that, we define "energy scalars" analogously to the Ricci scalars: $t_{00} \equiv 8 \pi \mathcal{T}_{11}$, and so on, such that Einstein's equation becomes

$$
\Phi_{i j}=t_{i j} \quad \text { and } \quad \Pi=t_{\Pi}
$$

with $i, j \in\{0,1,2\}$. For the Kerr-Newman solution, the only nonzero entry is $t_{11}$, which can be expressed in terms of a complex number $\varphi_{1}$ called a Maxwell scalar: ${ }^{19}$

$$
t_{11}=\left|\varphi_{1}\right|^{2}, \quad \varphi_{1}=\frac{Q}{\sqrt{2}\left(R^{*}\right)^{2}} .
$$

Our point is that the equation $t_{11}=\left|\varphi_{1}\right|^{2}$ is the statement $T_{\mu \nu}=F_{\mu \rho} F_{\nu}{ }^{\rho}-\frac{1}{4} g_{\mu \nu} F_{\rho \sigma} F^{\rho \sigma}$ in the tangent space, and the equation $\Phi_{11}=t_{11}$ is Einstein's equation in the tangent space. The typically stated relation $\Phi_{11}=\left|\varphi_{1}\right|^{2}$ combines both.

Having traipsed through the background geometry, we are now ready to perturb it.

\section{Shifted frame and Kerr-Schild form}

Relative to the standard frame of the Kerr-Newman background, and in terms of a general function $S(t, r, \theta, \varphi)$, we define the shifted fram $e^{20}$

$$
\tilde{l} \equiv l, \tilde{l}^{\prime} \equiv l^{\prime}+S l, \quad \tilde{m} \equiv m \text {. }
$$

It cannot be emphasized enough that the meaning of $\tilde{l}^{\prime}$ in components is

$$
\tilde{l}^{\prime}=\tilde{l}_{\mu}^{\prime} d x^{\mu}=\left(l_{\mu}^{\prime}+S l_{\mu}\right) d x^{\mu},
$$

not $\tilde{l}_{\mu}^{\prime} d \tilde{x}^{\mu}$ for some shifted coordinate basis $d \tilde{x}^{\mu}$. Otherwise the shift would describe a change of coordinates, not a physical perturbation.

\footnotetext{
${ }^{19}$ The Maxwell scalars are defined as the components of the electromagnetic curvature contracted with the vectors of the null frame:

$$
\varphi_{0} \equiv F_{\mu \nu} l^{\mu} m^{\nu}, \varphi_{1} \equiv \frac{1}{2} F_{\mu \nu}\left(l^{\mu} l^{\prime \nu}+m^{\prime \mu} m^{\nu}\right), \varphi_{2} \equiv F_{\mu \nu} m^{\prime \mu} l^{\prime \nu} .
$$

Note that because $F_{\mu \nu}=-F_{\nu \mu}$, we have $\varphi_{2}=-\varphi_{0}^{\prime}$ and $\varphi_{1}^{\prime}=-\varphi_{1}$.

${ }^{20}$ Only during revisions did we find the apropos work by Fels and Held [45]. While their shift is like ours, their analysis differs. Strikingly, they consider shifting Type D backgrounds but conclude that "as seeds they are not very fruitful." We disagree.
} 
Recalling Eq. (2.2), we define the shifted line element as

$$
d \tilde{s}^{2} \equiv-2 \tilde{l} \tilde{l}^{\prime}+2 \tilde{m} \tilde{m}^{\prime}=d s^{2}-2 S l l .
$$

Since we have chosen $l^{\mu}$ to be tangent to a shear-free geodesic congruence of the unshifted spacetime, the shifted line element is of the generalized Kerr-Schild form, as defined by Taub [30]. If we turn off the angular momentum and the charge and choose the ansatz

$$
S=\frac{\Delta}{2 r^{2}} \frac{U}{V} \delta(U) f(\theta, \varphi) \quad(a=Q=0)
$$

then we will reproduce exactly the Dray-'t Hooft metric [19]. If we turn off the angular momentum but leave the charge nonzero and use the same functional form for the ansatz, we will reproduce the metric of Alonso-Zamorano [22] and Sfetsos [23].

\subsection{From Reissner-Nordström to Kerr-Newman}

To generalize to a rotating background, we will scrutinize the factors that appear in Eq. (3.4).

First, by revisiting our conventions for the unshifted frame and staring at the definition of the shifted one, we conclude that the factor $\frac{\Delta}{2 r^{2}}$ compensates for the asymmetric normalization of $l_{\mu}$ relative to $l_{\mu}^{\prime}$. So the generalization of this factor to the rotating case is clear:

$$
\frac{\Delta}{2 r^{2}} \rightarrow \frac{\Delta}{2|R|^{2}} .
$$

Second, we have defined the Kruskal-like coordinates so that they mimic the coordinates in the nonrotating case: The future horizon is still at $U=0$, and the radial function $r$ depends only on the product $U V$. So we might hope that the factor $\frac{U}{V} \delta(U)$ could remain unmodified.

Third, we recognize that the function $f(\theta, \varphi)$ is defined only at the origin of Kruskal-like coordinates $(U=V=0)$. Extrapolating to the Kerr-Newman spacetime should therefore entail the generalization

$$
(\theta, \varphi) \rightarrow(\theta, \chi)
$$

This cross-examination of the Dray-'t Hooft solution coupled with the clear geometrical underpinning of the Newman-Penrose formalism led us to the conviction that the perturbed Kerr-Newman geometry should be described by the shifted frame in Eq. (3.1) with the following ansatz:

$$
S=\frac{\Delta}{2|R|^{2}} \frac{U}{V} \delta(U) f(\theta, \chi) .
$$

We will call $S$ the shift function, and we will call $f(\theta, \chi)$ the horizon field. When we calculate the curvature scalars, we will work directly with the rescaled frame in Eq. (2.48), thereby enlisting the rescaled shift function

$$
\hat{S}=(-U)^{-2} S=\frac{1}{2|R|^{2}} \frac{\Delta}{U V} \delta(U) f(\theta, \chi) .
$$


Like everything else in the hatted basis, this shift function is finite at the horizon.

By comparing the GHP representations $l_{\mu}^{\prime} \sim\left(-\frac{1}{2},-\frac{1}{2}\right)$ and $l_{\mu} \sim\left(+\frac{1}{2},+\frac{1}{2}\right)$ in the context of Eq. (3.1), we deduce that the shift function must transform as

$$
S \sim(-1,-1)
$$

When interpreting the formulas Eqs. (3.7) and (3.8) in the GHP formalism, we assign the horizon field $f(\theta, \chi)$ the weights of the shift function:

$$
f(\theta, \chi) \sim(-1,-1)
$$

The remaining factors are to be treated as ordinary functions, not physical degrees of freedom, and are therefore assigned weights $(0,0)$.

By explicit calculation, we will indeed find that the ansatz in Eq. (3.7) results in a shifted Ricci tensor of the form

$$
\tilde{R}_{\mu \nu}=R_{\mu \nu}+R_{U U}^{\mathrm{shift}} \delta_{\mu}^{U} \delta_{\nu}^{U}
$$

and therefore correctly generalizes the Dray-'t Hooft solution to a rotating background.

\subsection{Preliminary commentary}

Before focusing on $R_{U U}$, we wish to preview a miracle: If the unshifted frame is aligned with shear-free null geodesics $\left(\kappa=\sigma=\kappa^{\prime}=\sigma^{\prime}=0\right)$ and if the unshifted $\Phi_{00}$ is zero, the shifted Ricci tensor will depend only linearly on the shift function $S{ }^{21}$

We will proceed step by step through the spin coefficient formalism to understand why this happens. A practical reason is to derive master formulas for the spin coefficients and curvature scalars of generalized Kerr-Schild spacetimes. For the spin coefficients we will maintain full generality in the background, but for the curvature scalars we will restrict to shear-free geodesic congruences.

\subsection{Shifted spin coefficients}

By shifting both sides of the null Cartan equations [Eq. (2.32)] and solving them, we can express the shifted spin coefficients in terms of their unshifted values.

Start with the equation for $d l$, and tilde every term: 22

$$
d \tilde{l}=-2 \operatorname{Re}(\tilde{\varepsilon}) \tilde{l} \wedge \tilde{l}^{\prime}+2 i \operatorname{Im}(\tilde{\rho}) \tilde{m} \wedge \tilde{m}^{\prime}+\left[\left(\tilde{\tau}-\tilde{\beta}+\tilde{\beta}^{\prime *}\right) \tilde{m}^{\prime} \wedge \tilde{l}+\tilde{\kappa} \tilde{m}^{\prime} \wedge \tilde{l}^{\prime}+\text { c.c. }\right] .
$$

\footnotetext{
${ }^{21}$ This was in fact noticed by Taub $\sqrt[30]{ }$ and by Alonso and Zamorano 22 .

${ }^{22}$ Note that, in keeping with our advisory remark below the definition of the shifted frame [Eq. [3.1]], we do not tilde the exterior derivative operator.
} 
By inserting into the right-hand side the definition of the shifted frame in terms of the unshifted frame and recalling that $l \wedge l=0$, we find

$$
d \tilde{l}=-2 \operatorname{Re}(\tilde{\varepsilon}) l \wedge l^{\prime}+2 i \operatorname{Im}(\tilde{\rho}) m \wedge m^{\prime}+\left[\left(\tilde{\tau}-\tilde{\beta}+\tilde{\beta}^{\prime *}+\tilde{\kappa} S\right) m^{\prime} \wedge l+\tilde{\kappa} m^{\prime} \wedge l^{\prime}+\text { c.c. }\right] .
$$

Since $\tilde{l}=l$, we have $d \tilde{l}=d l$, so the left-hand side can be replaced with the untilded version of Eq. (3.12). The four basis 2-forms $l \wedge l^{\prime}, m \wedge m^{\prime}, m^{\prime} \wedge l$, and $m^{\prime} \wedge l^{\prime}$ are linearly independent, so we can match their coefficients on both sides to obtain the first set of shifted spin coefficient equations:

$$
\operatorname{Re}(\tilde{\varepsilon})=\operatorname{Re}(\varepsilon), \operatorname{Im}(\tilde{\rho})=\operatorname{Im}(\rho), \tilde{\tau}-\tilde{\beta}+\tilde{\beta}^{\prime *}+\tilde{\kappa} S=\tau-\beta+\beta^{\prime *}, \quad \tilde{\kappa}=\kappa .
$$

Next up, $d l^{\prime}$. The right-hand side parallels that for $d l$, but since $l^{\prime}=l^{\prime}+S l$ the left-hand side is more complicated. Not only do we require the untilded equations for both $d l^{\prime}$ and $d l$, we also require the exterior derivative of the shift function:

$$
d S \equiv d x^{\mu} \partial_{\mu} S=e^{a} \partial_{a} S=-l D^{\prime} S-l^{\prime} D S+m \delta^{\prime} S+m^{\prime} \delta S .
$$

Matching the coefficients of the basis 2-forms gives the second set of shifted spin coefficient equations:

$$
\begin{aligned}
& \operatorname{Re}\left(\tilde{\varepsilon}^{\prime}\right)=\operatorname{Re}\left(\varepsilon^{\prime}\right)-\operatorname{Re}(\varepsilon) S+\frac{1}{2} D S, \quad \operatorname{Im}\left(\tilde{\rho}^{\prime}\right)=\operatorname{Im}\left(\rho^{\prime}\right)-\operatorname{Im}(\rho) S, \\
& \tilde{\tau}^{\prime}-\tilde{\beta}^{\prime}+\tilde{\beta}^{*}=\tau^{\prime}-\beta^{\prime}+\beta^{*}+\kappa^{*} S, \quad \tilde{\kappa}^{\prime}+\left(\tilde{\tau}^{\prime}-\tilde{\beta}^{\prime}+\tilde{\beta}^{*}\right) S=\kappa^{\prime}+\left(\tau^{*}-\beta^{*}+\beta^{\prime}\right) S+\delta^{\prime} S .
\end{aligned}
$$

Before moving on, it is helpful to take stock of where we are. We have already solved directly for $\operatorname{Re}(\tilde{\varepsilon}), \operatorname{Im}(\tilde{\rho})$, and $\tilde{\kappa}$, and may thereby observe that they remain unshifted. We have also solved for $\operatorname{Re}\left(\tilde{\varepsilon}^{\prime}\right)$ and $\operatorname{Im}\left(\tilde{\rho}^{\prime}\right)$. By inserting the third equation in Eq. (3.16) into the fourth one, we obtain the shifted $\kappa^{\prime}$ :

$$
\tilde{\kappa}^{\prime}=\kappa^{\prime}+\left(\delta^{\prime}-2 \beta^{*}+2 \beta^{\prime}\right) S+\left(\tau^{*}-\tau^{\prime}\right) S-\kappa^{*} S^{2} .
$$

Recall that $S \sim(-1,-1)$ and that the GHP-covariant version of $\delta^{\prime}$ is $\delta^{\prime}=\delta^{\prime}-2 h \beta^{\prime}+2 h \beta^{*}$. As expected from GHP covariance, the NP derivatives and gauge fields appear in just the right combination to form a covariant derivative:

$$
\tilde{\kappa}^{\prime}=\kappa^{\prime}+ð^{\prime} S+\left(\tau^{*}-\tau^{\prime}\right) S-\kappa^{*} S^{2} .
$$

On the other hand, the terms involving $D, \varepsilon$, and $\varepsilon^{*}$ in $\operatorname{Re}\left(\tilde{\varepsilon}^{\prime}\right)$ do not collect themselves into a GHP-covariant combination. But that too is expected: While $\tilde{\kappa}^{\prime}$ is a weighted quantity, $\tilde{\varepsilon}^{\prime}$ is not. By solving the matrix inversion problem in Eq. (2.6) for the shifted frame, we obtain the shifted NP derivatives:

$$
\tilde{D}=D, \quad \tilde{D}^{\prime}=D^{\prime}-S D, \quad \tilde{\delta}=\delta
$$

We will see that $\tilde{\varepsilon}^{\prime}$ will in fact combine with $\tilde{D}^{\prime}$ to create a shifted $\tilde{\mathrm{p}}^{\prime}$ that can be written in terms of GHP-covariant quantities. But to prove that, we will need to solve for the shifted $\operatorname{Im}\left(\tilde{\varepsilon}^{\prime}\right)$, and for that we will need to study $d m$. 
Applying the above procedure to $d m$, we find the final set of shifted spin coefficient equations:

$$
\begin{aligned}
& \tilde{\beta}+\tilde{\beta}^{\prime *}=\beta+\beta^{\prime *}, \quad \tilde{\tau}-\tilde{\tau}^{* *}=\tau-\tau^{\prime *}, \quad \tilde{\rho}-2 i \operatorname{Im}(\tilde{\varepsilon})=\rho-2 i \operatorname{Im}(\varepsilon), \\
& \tilde{\rho}^{\prime}+2 i \operatorname{Im}\left(\tilde{\varepsilon}^{\prime}\right)+(\tilde{\rho}-2 i \operatorname{Im}(\tilde{\varepsilon})) S=\rho^{\prime}+2 i \operatorname{Im}\left(\varepsilon^{\prime}\right), \quad \tilde{\sigma}^{\prime *}+\tilde{\sigma} S=\sigma^{\prime *}
\end{aligned}
$$

By solving Eqs. (3.14), (3.16), and (3.20), we learn that the weighted spin coefficients and gauge field associated with $l_{\mu}$ do not receive corrections:

$$
\tilde{\kappa}=\kappa, \quad \tilde{\tau}=\tau, \quad \tilde{\sigma}=\sigma, \quad \tilde{\rho}=\rho, \quad \tilde{\varepsilon}=\varepsilon .
$$

While it should not be surprising that $\kappa, \sigma, \rho$, and $\varepsilon$ do not receive corrections, it may be unexpected that $\tau$ does not shift. It turns out that $\tau^{\prime}$ also remains unshifted:

$$
\tilde{\tau}^{\prime}=\tau^{\prime} .
$$

So the timelike expansion $\tau+\tau^{\prime *}$ and the timelike twist $\tau-\tau^{\prime *}$ remain unshifted.

The weighted spin coefficients and gauge field associated with $l_{\mu}^{\prime}$ do receive corrections:

$$
\begin{aligned}
& \tilde{\kappa}^{\prime}=\kappa^{\prime}+\left[\chi^{\prime}+\left(\tau^{*}-\tau^{\prime}\right)\right] S-\kappa^{*} S^{2}, \quad \tilde{\sigma}^{\prime}=\sigma^{\prime}-\sigma^{*} S, \quad \tilde{\rho}^{\prime}=\rho^{\prime}-\rho S, \\
& \tilde{\varepsilon}^{\prime}=\varepsilon^{\prime}-\varepsilon^{*} S+\frac{1}{2} D S-i \operatorname{Im}(\rho) S .
\end{aligned}
$$

In general, the transverse gauge fields also receive corrections:

$$
\tilde{\beta}=\beta+\frac{1}{2} \kappa S, \quad \tilde{\beta}^{\prime}=\beta^{\prime}-\frac{1}{2} \kappa^{*} S .
$$

From Eqs. (3.21)-(3.24) we conclude that if we align $l^{\mu}$ with background geodesics - namely if $\kappa=0$ - then not only do the formulas simplify considerably, but all nonlinearity in the shift function drops out of the spin coefficients.

This already implies $\tilde{R}_{a b c d}=R_{a b c d}+S R_{a b c d}^{(1)}+S^{2} R_{a b c d}^{(2)}$, i.e., there are no terms of $O\left(S^{3}\right)$ or higher. Furthermore, if the geodesics to which $l^{\mu}$ are aligned can also be taken shear-freenamely if $\sigma=0$ - then we get $\tilde{\sigma}^{\prime}=\sigma^{\prime}$ as well. Finally, if we also align $l^{\prime \mu}$ with background shear-free geodesics, then

$$
\tilde{\kappa}^{\prime}=\left[\chi^{\prime}+\left(\tau^{*}-\tau^{\prime}\right)\right] S, \quad \tilde{\rho}^{\prime}=\rho^{\prime}-\rho S, \quad \tilde{\varepsilon}^{\prime}=\varepsilon^{\prime}-\varepsilon^{*} S+\frac{1}{2} D S-i \operatorname{Im}(\rho) S .
$$

In this case, the only GHP-covariant derivative that shifts is $\mathrm{p}^{\prime}$. The shifted version acting on a function $f_{h, \bar{h}} \sim(h, \bar{h})$ is

$$
\tilde{\mathrm{p}}^{\prime} f_{h, \bar{h}}=\left[\mathrm{b}^{\prime}-S \mathrm{~b}-(h+\bar{h})(\mathrm{b} S)+2 i(h-\bar{h}) \operatorname{Im}(\rho) S\right] f_{h, \bar{h}} .
$$

This vindicates the discussion below Eq. (3.18) and completes our derivation of the shifted spin coefficients.

Dray and 't Hooft explained [19] that test particles crossing the shockwave get translated and refracted. (See also the work by Matzner [46].) In the spin coefficient formalism, these effects are described by the shifted versions of $\rho^{\prime}$ and $\kappa^{\prime}$ - to the physics we now turn. 


\subsection{Shifted horizon}

Cartography of the horizon requires the hatted basis. As we discussed back in Sec. 2.8, the future horizon can be defined locally as the subspace of Kruskal-like coordinates on which the expansion of the outgoing congruence vanishes $(\hat{\rho}=0)$. Similarly, the past horizon is the subspace on which the expansion of the ingoing congruence vanishes $\left(\hat{\rho}^{\prime}=0\right)$.

Recalling the unshifted $\hat{\rho}$ and $\hat{\rho}^{\prime}$ from Eq. 2.45 and the shift described in Eq. (3.25), we find that the coordinate $V$ receives a correction while the coordinate $U$ does not:

$$
\begin{aligned}
& \tilde{\hat{\rho}}=\hat{\rho} \Longrightarrow \tilde{U}=U, \\
& \tilde{\hat{\rho}}^{\prime}=\hat{\rho}^{\prime}-\hat{\rho} \hat{S}=\left(1+\frac{U}{V} \delta(U) f(\theta, \chi)\right) \hat{\rho}^{\prime} \equiv\left[\frac{1}{2|R|^{2}}\left(\frac{\Delta}{U V}\right) \frac{1}{R^{*}}\right] \tilde{V} \\
& \Longrightarrow \tilde{V}-V=U \delta(U) f(\theta, \chi) .
\end{aligned}
$$

This last expression implies that smooth functions of $U$ will experience no coordinate shift, while functions that go as $\frac{1}{U}$ near $U=0$ will experience a discontinuity in the coordinate. To see this, interpret Eq. (3.27) as a differential equation in $U$ in the vicinity of $U=0$, i.e., $\frac{d(\tilde{V}-V)}{d U}=\lim _{U \rightarrow 0} \frac{\tilde{V}-V}{U}=\delta(U) f(\theta, \chi)$. Integration then gives

$$
\tilde{V}=V+\Theta(U) f(\theta, \chi) .
$$

This is the shift as described by Dray and 't Hooft [19] and by Sfetsos [23].

\subsection{Refraction}

Since every acolyte of Penrose knows that $\kappa$ and $\kappa^{\prime}$ describe the refraction of light rays, the result that $\kappa^{\prime}$ becomes nonzero after the shift speaks for itself.

\section{Petrov classification for the Kerr-Newman shockwave}

Let the games begin. We will first shift the Weyl scalar $\Psi_{4} \sim(-2,0)$, or more conveniently its complex conjugate $\Psi_{4}^{*} \sim(0,-2)$. Since this is just our opening act, we will reserve intricate computational details for the main event, the shifted Ricci scalars.

\subsection{Shifted $\Psi_{4}$ and physical interpretation}

Aligning the background frame with shear-free geodesic congruences but assuming an arbitrary shift function $S$, we find:

$$
\tilde{\Psi}_{4}^{*}=\Psi_{4}^{*}+\partial ð S+2 \tau ð S .
$$

To obtain this we used the complex conjugate and the prime of $\Phi_{02}$ from Eq. (2.57) in the forms $\chi^{\prime} \tau^{*}=-\tau^{* 2}$ and $\chi^{\prime} \tau^{\prime}=-\tau^{\prime 2}$, which hold when $\Phi_{02}=0$.

To specialize to the shockwave, hat everything and insert the ansatz of Eq. (3.8) for the 
shift function. Since the calculation is laborious, it is advantageous to first enumerate conceivable terms.

Remember that the horizon field $f(\theta, \chi)$ has weights $(-1,-1)$. Since $\Psi_{4}^{*}$ has weights $(0,-2)$, we will have to find operators of weights $(1,-1)$. Fortunately, the list of such operators that are nonzero at the Kerr-Newman horizon is short:

$$
\text { ðð ; } \tau \text { ð }, \tau^{* ð} ; \tau^{2}, \tau^{* 2}, \tau \tau^{*} .
$$

In principle we would also need $\partial \tau$ and $\partial \tau^{\prime *}$, but again when $\Phi_{02}=0$ those can be traded for $-\tau^{2}$ and $-\tau^{* 2}$. So the result must have the form

$$
\hat{\tilde{\Psi}}_{4}^{*}=k_{0} \delta(U)\left[ð ð f+\left(k_{1} \tau+k_{2} \tau^{*}\right) ð f+\left(k_{3} \tau^{2}+k_{4} \tau^{* 2}+k_{5} \tau \tau^{*}\right) f\right]
$$

for some functions $k_{i}(\theta)$ that will depend on the parameters $r_{+}, a$, and $\alpha$. Whether by hand or by machine we ultimately find:

$$
\begin{aligned}
& k_{0}=-\frac{c}{2|R|^{2}}, \quad k_{1}=\frac{\alpha|R|^{2}}{r}, \quad k_{2}=-2\left(1+\frac{\alpha|R|^{2}}{2 r}\right), \quad k_{3}=\left(\frac{\alpha|R|^{2}}{2 r}\right)^{2}, \\
& k_{4}=1+\left(1+\frac{\alpha|R|^{2}}{2 r}\right)^{2}, \quad k_{5}=-\frac{\alpha|R|^{2}}{r}\left(1+\frac{\alpha|R|^{2}}{2 r}\right) .
\end{aligned}
$$

On the way to this result, we encounter terms involving $\partial_{U} \delta(U)$ and $\partial_{U}^{2} \delta(U) .{ }^{23}$ We interpret them according to the distributional edict of integrating by parts against an arbitrary smooth test function $\mathcal{F}(U)$ :

$$
\int d U \mathcal{F}(U) \partial_{U}^{n} \delta(U)=\int d U(-1)^{n} \partial_{U}^{n} \mathcal{F}(U) \delta(U)
$$

It should also be understood, as required by the overall factor $\delta(U)$, that all instances of $r$ in Eq. (4.4) actually denote $r_{+}$. Also note that numerically we have

$$
\tau^{\prime}=-\frac{R}{R^{*}} \tau^{*}
$$

so it is possible to shuffle terms among the coefficients $k_{3}, k_{4}$, and $k_{5}$. The particular form shown in Eq. (4.4) is what we exhumed upon performing the rituals to be disclosed in Sec. 6 .

Invoking the gravitational compass from Sec. 2.11, we interpret Eqs. (4.3) and (4.4) as describing a transverse "outgoing" gravitational wave stuck to the horizon. 24

\footnotetext{
${ }^{23}$ We also stumble upon the gargantuan notational implosion " $\partial \delta(U)=\delta \delta(U)$."

${ }^{24}$ We cannot help calling the reader's attention to the following famous quotation: "Now, here, you see, it takes all the running you can do, to keep in the same place." This is originally from Through the Looking-Glass by Lewis Carroll, but we first encountered its application to the horizon of a black hole from the textbook on the Kerr geometry by O'Neill [47].
} 


\subsection{Nonrotating limit}

It is worth pausing to consider the nonrotating limit, $a \rightarrow 0$, in which case only the ðð $f$ term in Eq. (4.3) survives.

As far as we know, the Weyl scalars for the shifted Reissner-Nordström geometry have not been calculated explicitly, so we will unpack the definitions of the GHP derivatives at the horizon. Remembering that $f \sim(-1,-1)$ and therefore $\partial f \sim\left(-\frac{1}{2},-\frac{3}{2}\right)$, and that in the nonrotating limit we have $\beta=\beta^{\prime}=\beta^{*}=\beta^{\prime *}$, we find:

$$
\text { ðð }\left.f\right|_{a=0}=\delta \delta f-2 \beta \delta f .
$$

\subsection{Shifted $\Psi_{3}$ and Petrov type}

Our debt to $\Psi_{4}$ settled, we turn to $\Psi_{3}$. Shifting the frame (with $\kappa=\sigma=\kappa^{\prime}=\sigma^{\prime}$ ) seemingly produces this Weyl scalar:

$$
\tilde{\Psi}_{3}^{*}=\Psi_{3}^{*}+\frac{1}{4}(\mathrm{~b} ð+ð \mathrm{p}) S+\frac{1}{4}\left(2 \tau-\tau^{\prime *}\right) \mathrm{p} S+\frac{1}{4}\left(2 \rho-5 \rho^{*}\right) ð S-\frac{1}{2}\left[\left(2 \tau-\tau^{\prime *}\right) \rho^{*}+ð \rho^{*}\right] S .
$$

But by hatting and specializing to Eq. (3.8), we find that each term in Eq. (4.8) goes to zero at $U=0$ for fixed nonzero $V$ :

$$
\hat{\tilde{\Psi}}_{3}^{*}=0 \text {. }
$$

Since the unshifted geometry already had a nonzero $\Psi_{2}$, we conclude that the shockwave is Petrov type II:

$$
\hat{\tilde{\Psi}}_{0}=\hat{\tilde{\Psi}}_{1}=\hat{\tilde{\Psi}}_{3}=0, \quad \hat{\tilde{\Psi}}_{2} \neq 0, \quad \hat{\tilde{\Psi}}_{4} \neq 0 .
$$

To quote Szekeres: "[I]t can be viewed as a Coulomb field with an outgoing wave component superimposed" 42].

\subsection{Curvatures of submanifolds}

Shifting both sides of the GHP commutator equations [see Eq. (2.49]), we find

$$
\tilde{\mathcal{K}}=\mathcal{K}+\operatorname{Im}(\rho)[2 \operatorname{Im}(\rho) S+i(\mathrm{p} S)], \quad \tilde{\mathcal{K}}_{s}=\mathcal{K}_{s}-\frac{1}{2}\left(\mathrm{p}^{2} S\right)+i \mathrm{p}[\operatorname{Im}(\rho) S] .
$$

But if we hat everything and specialize to the shockwave ansatz, we will find that all of the corrections in Eq. 4.11) go to zero. Curiously enough, the shockwave does not alter the spacelike and timelike curvatures.

\subsection{Shifted $\Psi_{2}$}

Inserting Eq. 4.11) into Eq. (2.67) provides the shifted Weyl scalar of weight zero:

$$
\tilde{\Psi}_{2}=\Psi_{2}+\frac{1}{6} \mathrm{p}^{2} S-\frac{1}{3}\left(2 \rho-\rho^{*}\right) \mathrm{p} S+\frac{1}{3}\left[\left(3 \rho-2 \rho^{*}\right) \rho+\Phi_{00}\right] S .
$$


To arrive at this expression, we used the relation 25

$$
\Phi_{00}=-\left(\mathrm{b} \rho+\rho^{2}\right) \quad(\text { if } \kappa=\sigma=0)
$$

along with $\Phi_{00}^{*}=\Phi_{00}$. Just as we found for the shifted $\Psi_{3}$, we find upon disbursing hats and availing ourselves of Eq. (3.8) that the correction to $\Psi_{2}$ is zero.

Appealing again to the gravitational compass [42], we say that the Coulomb field remains unchanged by the presence of a massless particle on the future horizon.

\section{$5 \quad$ Shifted Ricci scalars}

Show time. We will first present the shifted Ricci scalars for the generalized Kerr-Schild geometry under the assumption $\kappa=\kappa^{\prime}=\sigma=\sigma^{\prime}$, and then we will specialize to the shockwave.

\subsection{Ricci scalar of weight $(-1,-1)$ : Absence of nonlinearity}

After the shift from Eq. (3.1), three of the Ricci scalars will become nonzero. Of these, the apple of our eye will be $\Phi_{22} \sim(-1,-1)$.

This quantity is defined by priming the definition of $\Phi_{00}$ in Eq. (2.57):

$$
\Phi_{22}=-\left(\mathrm{p}^{\prime} \rho^{\prime}+\rho^{\prime 2}\right)+ð \kappa^{\prime}+\tau \kappa^{\prime}+\tau^{\prime} \kappa^{\prime *}-\left|\sigma^{\prime}\right|^{2} .
$$

Using the shifted $\rho^{\prime}$ from Eq. (3.25) and the shifted $\mathrm{p}^{\prime}$ from Eq. (3.26), and using $h=\bar{h}=-\frac{1}{2}$ for $\rho^{\prime}$ [recall Eq. (2.27)], we find:

$$
\begin{aligned}
& \tilde{\mathrm{p}}^{\prime} \tilde{\rho}^{\prime}=\mathrm{p}^{\prime} \rho^{\prime}+\left(\rho^{\prime} \mathrm{p}-\rho \mathrm{p}^{\prime}\right) S-\left(\mathrm{p}^{\prime} \rho+\mathrm{p} \rho^{\prime}\right) S+(\mathrm{b} \rho) S^{2} \\
& \tilde{\rho}^{\prime 2}=\rho^{\prime 2}-2 \rho \rho^{\prime} S+\rho^{2} S^{2}
\end{aligned}
$$

It is worth keeping in mind the formula for $\Phi_{00}$ under the shear-free geodesic assumption [Eq. 4.13)]. Next, for $\sigma \sigma^{\prime}=\kappa \kappa^{\prime}=0$, we have:26

$$
\begin{aligned}
\Psi_{2}+2 \Pi & =-\left(\nearrow^{\prime} \tau+|\tau|^{2}\right)+\mathrm{p}^{\prime} \rho+\rho^{\prime *} \rho \\
& =-\left(ð \tau^{\prime}+\left|\tau^{\prime}\right|^{2}\right)+\mathrm{p} \rho^{\prime}+\rho^{*} \rho^{\prime} .
\end{aligned}
$$

It is a matter of some discretion which variables to keep and which to trade away. We are guided by comparison with the nonrotating limit, which suggests we should express as much as possible in terms of $\tau$ and $\tau^{\prime}$ and their derivatives. So we will use Eqs. (5.3) and (5.4) to evict $\mathrm{p}^{\prime} \rho$ and $\mathrm{p} \rho^{\prime}$ from Eq. (5.2).

\footnotetext{
${ }^{25}$ This is Raychaudhuri's equation for null shear-free geodesic congruences. When $\Phi_{00}=0$, it tells us that $\mathrm{b} \rho=-\rho^{2}$. Given the standard interpretation of $\operatorname{Re}(\rho)$ as the expansion, we recognize this as the focusing theorem.

${ }^{26}$ Attentive readers have every right to be confused by the second equality: Indeed it turns out that the combination of derivatives and products of spin coefficients in Eq. (5.3) equals its primed version in Eq. (5.4). This must be so, since both $\Psi_{2}=C_{1342}$ and $\Pi=\frac{1}{12}\left(R_{12}-R_{34}\right)$ are self-prime.
} 
With our shifted $\kappa^{\prime}$ from Eq. (3.25), we find:

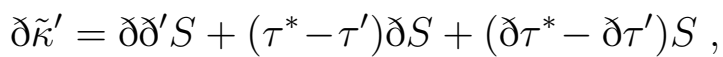

$$
\begin{aligned}
& \tau \tilde{\kappa}^{\prime}+\tau^{\prime} \tilde{\kappa}^{\prime *}=\left(\tau ð^{\prime}+\tau^{\prime} ð\right) S+\left(|\tau|^{2}-\left|\tau^{\prime}\right|^{2}\right) S .
\end{aligned}
$$

Eqs. (5.2)-(5.5) then supply the preliminary expression:

$$
\begin{aligned}
\tilde{\Phi}_{22} & =\Phi_{22}+\left(\rho \mathrm{p}^{\prime}-\rho^{\prime} \mathrm{p}\right) S+\Phi_{00} S^{2}+\chi^{\prime} S+\tau{ }^{\prime} S+\tau^{*} \text { } S \\
& +\left[2 \rho \rho^{\prime}-\left(\rho \rho^{\prime *}+\rho^{*} \rho^{\prime}\right)+\partial^{\prime} \tau+ð \tau^{*}+2|\tau|^{2}+2\left(\Psi_{2}+2 \Pi\right)\right] S .
\end{aligned}
$$

Behold: For a background in which $\Phi_{00}=0$, all nonlinear dependence on the perturbation drops out of the curvature scalars. Terms of $O\left(S^{2}\right)$ could not possibly show up elsewhere, because the only curvature scalar with the appropriate weight to include a product of shifted quantities (in this case $\mathrm{p}^{\prime}$ and $\rho^{\prime}$ ) is $\Phi_{22}$.

To make sense of Eq. (5.6) we will rewrite it in a manifestly real form:

$$
\begin{aligned}
& \tilde{\Phi}_{22}=\operatorname{Re}\left(\tilde{\Phi}_{22}\right)=\Phi_{22}+\operatorname{Re}\left[\left(\rho \mathrm{p}^{\prime}-\rho^{\prime} \mathrm{p}\right) S\right]+\Phi_{00} S^{2} \\
& +\frac{1}{2}\left[\chi^{\prime}+\chi^{\prime} \partial+\left(\tau+\tau^{* *}\right) ð^{\prime}+\left(\tau^{*}+\tau^{\prime}\right) ð+\left(\tau-\tau^{* *}\right) ð^{\prime}+\left(\tau^{*}-\tau^{\prime}\right) ð\right] S \\
& +\left\{\left(\rho-\rho^{*}\right)\left(\rho^{\prime}-\rho^{*}\right)+\left(\chi^{\prime} \tau+\text { c.c. }\right)+2|\tau|^{2}+2\left[\operatorname{Re}\left(\Psi_{2}\right)+2 \Pi\right]\right\} S \text {. }
\end{aligned}
$$

Experts in the compacted formalism should recognize the combination $\partial \chi^{\prime}+\left(\tau+\tau^{\prime *}\right) ð^{\prime}+$ c.c. as part of the generalized Laplacian (we will get to this in Sec. 6). Before elaborating on this, we will vanquish the remaining curvature scalars.

\subsection{Other Ricci scalars}

The Ricci scalar of weight $(-1,0)$ is corrected by the general shift:27

$$
\tilde{\Phi}_{21}=\Phi_{21}+\frac{1}{4}\left(\mathrm{~b} \chi^{\prime}+\chi^{\prime} \mathrm{p}\right) S+\frac{1}{4}\left(2 \tau^{*}-\tau^{\prime}\right) \mathrm{p} S+\frac{1}{4}\left(3 \rho-2 \rho^{*}\right) ð^{\prime} S+\frac{1}{2}\left(\tau^{\prime} \rho-2 \tau^{*} \rho^{*}+ð^{\prime} \rho\right) S .
$$

For the Kerr-Newman background, we have $\Phi_{21}=0$. After hatting and specializing to Eq. (3.8), we find that each would-be contribution from $S$ to Eq. (5.8) is zero.

Next we have the Ricci scalar of weight $(0,0)$ :

$$
\tilde{\Phi}_{11}=\Phi_{11}+\frac{1}{4} \mathrm{p}^{2} S-\frac{1}{2}\left[|\rho|^{2}+\left(\rho-\rho^{*}\right)^{2}\right] S .
$$

Here too we find no correction to the unshifted value after hatting both sides of the equation and specializing to the shockwave: $\hat{\tilde{\Phi}}_{11}=\hat{\Phi}_{11}=\Phi_{11}$.

The Einstein-Hilbert curvature also superficially becomes nonzero as a result of the shift:

$$
\tilde{\Pi}=\Pi-\frac{1}{6}\left\{\frac{1}{2} \mathrm{p}^{2} S+\left(\rho+\rho^{*}\right) \mathrm{p} S+\left(|\rho|^{2}-2 \Phi_{00}\right) S\right\} .
$$

\footnotetext{
${ }^{27}$ The steps leading to this expression parallel closely those that led to $\tilde{\Psi}_{3}^{*}$.
} 
But we know that $\Pi$ is proportional to the Lagrangian of general relativity, so its first order variation must comport with the standard formula

$$
S_{\mathrm{GR}}[g+h]-S_{\mathrm{GR}}[g]=\frac{1}{2} \int d^{4} x|\operatorname{det}(g)|^{1 / 2} T^{\mu \nu} h_{\mu \nu}+O\left(h^{2}\right) .
$$

The shift from Eq. (3.1) effects the metric variation

$$
h_{\mu \nu}=-2 S l_{\mu} l_{\nu} .
$$

So varying the action with respect to $S$ will result in something proportional to $T^{\mu \nu} l_{\mu} l_{\nu}=$ $\mathcal{T}^{\mu \nu} l_{\mu} l_{\nu}=(8 \pi)^{-1} t_{00}$ (recall Sec. 2.12). Because the only nonzero energy scalar for the background spacetime is $t_{11} \propto\left(l_{\mu} l_{\nu}^{\prime}+l_{\mu}^{\prime} l_{\nu}+m_{\mu} m_{\nu}^{\prime}+m_{\mu}^{\prime} m_{\nu}\right) \mathcal{T}^{\mu \nu}$, we know that $t_{00}=0$ and thereby expect the $O(S)$ term in Eq. (5.11) to equal zero. ${ }^{28}$

The nonzero $O(S)$ term in Eq. (5.10) might invite consternation, but we have been cavalierly ignoring possible boundary terms in the action. So all we require is that the $O(h)$ term in Eq. (5.11) should be zero, not necessarily that the shift in $\Pi$ itself should be zero.

For Kerr-Newman, we have $e^{29}|\operatorname{det}(g)|^{1 / 2}=i \varepsilon^{\mu \nu \rho \sigma} l_{\mu} l_{\nu}^{\prime} m_{\rho} m_{\sigma}^{\prime}=|R|^{2} \sin \theta$. After integrating by parts, dropping total derivatives, and using $D$ and $\rho$ from Eqs. (2.8) and (2.33), we indeed obtain

$$
\int d^{4} x|\operatorname{det}(g)|^{1 / 2} \tilde{\Pi}=0
$$

This completes our account of the shifted curvature scalars for the generalized Kerr-Schild geometry. (The Ricci scalars not explicitly enumerated in this section do not shift.) Now we will specialize the shifted $\Phi_{22}$ to the shockwave.

\section{Derivatives of the shift}

The spacetime Laplacian $\nabla^{2}=\nabla_{\mu} \nabla^{\mu}$ finds refuge in the compacted spin coefficient formalism within a more general operator

$$
\square=-\square_{\|}+\square_{\perp}
$$

where

$$
\square_{\|} \equiv[\mathrm{p}+2 \operatorname{Re}(\rho)] \mathrm{p}^{\prime}+{ }^{\prime}, \square_{\perp} \equiv\left[ð+\left(\tau+\tau^{\prime *}\right)\right] \mathrm{\gamma}^{\prime}+{ }^{\prime} .
$$

The operator $\square_{\perp}$ will be called the "transverse box." Evaluating its action on the shift function is the most technically cumbersome aspect of computing $\tilde{\Phi}_{22}$.

We will do our best to show how the sausage is made without belaboring mindless algebra.

\footnotetext{
${ }^{28}$ We thank Alexei Kitaev for suggesting this check on our work.

${ }^{29}$ This expression for $|\operatorname{det}(g)|^{1 / 2}$ makes clear that it does not receive a correction from Eq. 3.1 .
} 


\subsection{Key facts}

To set up the calculation we will first collect some useful formulas.

From what may seem like a lifetime ago, we recall that $U \partial_{U} r=V \partial_{V} r$ (which can be traced back to the relation $\left.-U \partial_{U}+V \partial_{V}=\frac{1}{\alpha} \partial_{t}\right)$. Therefore, acting on a weight- $(0,0)$ function $F(r)$, we have:

$$
ð F(r)=0 .
$$

This is our first key fact.

Next we recall the explicit formulas for the timelike expansion and the timelike twist [Eq. (2.34)]. They will compose our basic mnemonic for making sense of complicated algebraic expressions: The trigonometric functions $\sin (2 \theta)$ and $\sin \theta$ should evoke $\tau+\tau^{\prime *}$ and $\tau-\tau^{\prime *}$ respectively.

We will use this to establish additional useful formulas. Treating $\delta(U)$ as having weight $(0,0)$ and summoning the NP derivatives in Kruskal-like coordinates [Eq. (2.48]), we find:

$$
ð \delta(U)=-\alpha \frac{i a \sin \theta}{R \sqrt{2}} U \partial_{U} \delta(U)=-\frac{\alpha|R|^{2}}{2 r}\left(\tau-\tau^{*}\right) U \partial_{U} \delta(U) .
$$

This is our second key fact.

Finally, we must bear in mind that although functions of $r$ can be treated as constants, the generalized radial function $R=r+i a \cos \theta$ is also a function of $\theta$. Treating this too as a function of weight $(0,0)$, we compute the following:

$$
\text { ð }\left(\frac{1}{|R|^{2}}\right)=-\frac{1}{\left(|R|^{2}\right)^{2}} \partial\left(|R|^{2}\right)=+\frac{a^{2} \sin (2 \theta)}{\sqrt{2} R|R|^{4}}=-\frac{1}{|R|^{2}}\left(\tau+\tau^{\prime *}\right) .
$$

This is our third key fact.

\subsection{Integration by parts}

We described back in Eq. 4.5 the standard integration-by-parts procedure that defines the delta function. Here it will be useful to study two special cases of that formula.

First consider a distribution $\mathcal{O}(U) U \partial_{U} \delta(U)$ (where the conditions on $\mathcal{O}(U)$ will be specified shortly), and integrate it against a test function $\mathcal{F}(U)$ that falls off quickly enough to merit dropping the boundary term:

$$
\int d U \mathcal{O}(U) U \partial_{U} \delta(U) \mathcal{F}(U)=-\int d U\left[\mathcal{O}(U) \mathcal{F}(U)+U \partial_{U}(\mathcal{O}(U) \mathcal{F}(U))\right] \delta(U) .
$$

If $\partial_{U}(\mathcal{O}(U) \mathcal{F}(U)) \sim U^{n}$ with $n \geq 0$ near $U=0$, then the second term evaluates to zero. We then obtain the following distributional equality:

$$
\mathcal{O}(U) U \partial_{U} \delta(U)=-\mathcal{O}(U) \delta(U)
$$


Along similar lines, we will obtain a second distributional equality:

$$
\mathcal{O}(U) U \partial_{U}\left(U \partial_{U} \delta(U)\right)=+\mathcal{O}(U) \delta(U)
$$

Equipped with the key facts in Eqs. (6.3)- 6.5 and the above distributional equalities, we are ready to face the transverse box.

\subsection{First-derivative terms}

We warm up with a first-derivative term. Specializing to the shockwave ansatz in Eq. (3.8) and applying our key facts, we obtain the preliminary expression

$$
\begin{aligned}
\partial \hat{S} & =\frac{1}{2} \frac{\Delta}{U V}\left[\partial\left(\frac{1}{|R|^{2}}\right) \delta(U) f(\theta, \chi)+\frac{1}{|R|^{2}} \partial \delta(U) f(\theta, \chi)+\frac{1}{|R|^{2}} \delta(U) ð f(\theta, \chi)\right] \\
& =\frac{1}{2|R|^{2}} \frac{\Delta}{U V}\left\{\delta(U)\left[\partial-\left(\tau+\tau^{\prime *}\right)\right] f(\theta, \chi)-\frac{\alpha|R|^{2}}{2 r}\left(\tau-\tau^{* *}\right) U \partial_{U} \delta(U) f(\theta, \chi)\right\} .
\end{aligned}
$$

Before integrating by parts against a test function, we need to multiply by $\tau^{*}+\tau^{\prime}$ to obtain the term $\left(\tau^{*}+\tau^{\prime}\right) \partial \hat{S}$ that appears in the transverse box. ${ }^{30}$

Note that since $|\tau|^{2}=\left|\tau^{\prime}\right|^{2}$ for the Kerr-Newman spacetime, we have

$$
\left(\tau^{*}+\tau^{\prime}\right)\left(\tau-\tau^{*}\right)=2 i \operatorname{Im}\left(\tau \tau^{\prime}\right)
$$

Using this and the distributional equality in Eq. (6.7), we obtain [also recall $c \equiv-\left.\frac{\Delta}{U V}\right|_{r=r_{+}}$ from Eq. 2.38]

$$
\left(\tau^{*}+\tau^{\prime}\right) \partial \hat{S}=-\frac{c}{2|R|^{2}} \delta(U)\left\{\left(\tau^{*}+\tau^{\prime}\right) \partial-\left|\tau+\tau^{* *}\right|^{2}+i \frac{\alpha|R|^{2}}{r} \operatorname{Im}\left(\tau \tau^{\prime}\right)\right\} f(\theta, \chi) \text {. }
$$

\subsection{Second-derivative terms}

Returning to Eq. (6.9), we act with $ð^{\prime}$ (and skip a few steps now that the method is presumably clear) to obtain

$$
\begin{aligned}
\chi^{\prime} \partial \hat{S} & =\frac{1}{2|R|^{2}} \frac{\Delta}{U V}\left\{\delta(U) ð^{\prime} \partial f-\left[\left(\tau+\tau^{\prime *}\right) \delta(U)+\frac{\alpha|R|^{2}}{2 r}\left(\tau-\tau^{\prime *}\right) U \partial_{U} \delta(U)\right] \partial^{\prime} f\right. \\
& \left.-\left[\left(\tau^{*}+\tau^{\prime}\right) \delta(U)+\frac{\alpha|R|^{2}}{2 r}\left(\tau^{*}-\tau^{\prime}\right) U \partial_{U} \delta(U)\right] ð f+\mathscr{C} f\right\},
\end{aligned}
$$

where

$$
\begin{aligned}
\mathscr{C} & =\left|\tau+\tau^{*}\right|^{2} \delta(U)-i \frac{\alpha|R|^{2}}{r} \operatorname{Im}\left(\tau \tau^{\prime}\right) U \partial_{U} \delta(U)-\left(\delta(U)+\frac{\alpha|R|^{2}}{2 r} U \partial_{U} \delta(U)\right) \partial^{\prime} \tau \\
& -\left(\delta(U)-\frac{\alpha|R|^{2}}{2 r} U \partial_{U} \delta(U)\right)\left(\partial \tau^{\prime}\right)^{*}+\left(\frac{\alpha|R|^{2}}{2 r}\right)^{2}\left|\tau-\tau^{* *}\right|^{2} U \partial_{U}\left[U \partial_{U} \delta(U)\right] .
\end{aligned}
$$

\footnotetext{
${ }^{30}$ Since $\tau^{*}+\tau^{\prime}$ depends on $U$ and $V$ only through $r=r(U V)$, and since we have already said such functions can be treated as constants with respect to $U \partial_{U}$ for our calculation, it does not matter in this particular instance whether we integrate by parts before or after multiplying by $\tau^{*}+\tau^{\prime}$.
} 
Note that in Eq. 6.12) the coefficient of $\partial^{\prime} f$ is the complex conjugate of the coefficient of $\partial f$. This did not have to be so, because we are computing $\chi^{\prime} \partial \hat{S}$ right now, not $\chi^{\prime} \partial \hat{S}+$ c.c., and in general $\chi^{\prime} \partial \neq \partial^{\prime}$.

This quantity ${ }^{\prime} \partial \hat{S}$ will be integrated directly against a test function (because it appears directly in the transverse box, which in turn appears directly in $\left.\hat{\tilde{\Phi}}_{22}\right)$, so we can use the distributional equalities in Eqs. (6.7) and (6.8), loosely expressed as $U \partial_{U} \delta(U) \rightarrow-\delta(U)$ and $U \partial_{U}\left[U \partial_{U} \delta(U)\right] \rightarrow+\delta(U)$. Applying these to Eq. 6.12), we obtain:

$$
\begin{aligned}
& \partial^{\prime} \partial \hat{S}=-\frac{c}{2|R|^{2}} \delta(U)\left\{\partial^{\prime} \partial-\left[\left(\tau+\tau^{\prime *}\right)-\frac{\alpha|R|^{2}}{2 r}\left(\tau-\tau^{\prime *}\right)\right] \partial^{\prime}-\left[\left(\tau^{*}+\tau^{\prime}\right)-\frac{\alpha|R|^{2}}{2 r}\left(\tau^{*}-\tau^{\prime}\right)\right] ð\right. \\
& \left.+\left|\tau+\tau^{\prime *}\right|^{2}+i \frac{\alpha|R|^{2}}{r} \operatorname{Im}\left(\tau \tau^{\prime}\right)-\left(1-\frac{\alpha|R|^{2}}{2 r}\right) \partial^{\prime} \tau-\left(1+\frac{\alpha|R|^{2}}{2 r}\right)\left(ð \tau^{\prime}\right)^{*}+\left(\frac{\alpha|R|^{2}}{2 r}\right)^{2}\left|\tau-\tau^{\prime *}\right|^{2}\right\} f(\theta, \chi) .
\end{aligned}
$$

\subsection{Transverse box}

Now we can finish the job. Returning to the first-derivative term in Eq. (6.9) and adding its complex conjugate, we obtain:

$$
\left(\tau^{*}+\tau^{\prime}\right) \partial \hat{S}+\text { c.c. }=-\frac{c}{2|R|^{2}} \delta(U)\left\{\left(\tau+\tau^{\prime *}\right) ð^{\prime}+\left(\tau^{*}+\tau^{\prime}\right) \partial-2\left|\tau+\tau^{\prime *}\right|^{2}\right\} f(\theta, \chi) .
$$

Next we obtain the anticommutator of GHP derivatives by taking Eq. 6.12 plus its complex conjugate:

$$
\begin{aligned}
& \chi^{\prime} \partial \hat{S}+\text { c.c. }=-\frac{c}{2|R|^{2}} \delta(U)\left\{\left(\chi^{\prime} \partial+\chi^{\prime}\right)+\left[-\left(2\left(\tau+\tau^{\prime *}\right)-\frac{\alpha|R|^{2}}{r}\left(\tau-\tau^{\prime *}\right)\right) \chi^{\prime}+\text { c.c. }\right]\right. \\
& \left.+2\left|\tau+\tau^{\prime *}\right|^{2}-\left(1-\frac{\alpha|R|^{2}}{2 r}\right)\left(ð^{\prime} \tau+\text { c.c. }\right)-\left(1+\frac{\alpha|R|^{2}}{2 r}\right)\left(ð \tau^{\prime}+\text { c.c. }\right)+2\left(\frac{\alpha|R|^{2}}{2 r}\right)^{2}\left|\tau-\tau^{\prime *}\right|^{2}\right\} f(\theta, \chi) \text {. }
\end{aligned}
$$

We then add Eqs. (6.15) and (6.16) to obtain the transverse box. For reasons morally unbeknownst to us, the $\left|\tau+\tau^{*}\right|^{2}$ term will cancel out. Also, for Kerr-Newman, we have

$$
\partial \tau^{\prime}=\partial^{\prime} \tau \text {. }
$$

There is probably a good reason for this, but it escapes us. At any rate, it implies that the $\alpha$-dependent parts of the coefficients of $\partial^{\prime} \tau+$ c.c. and $\partial \tau^{\prime}+$ c.c. drop out.

Therefore, the transverse box acting on the shift function, expressed in terms of GHP derivatives at the horizon, simplifies to:

$$
\begin{aligned}
& \square_{\perp} \hat{S}=-\frac{c}{2|R|^{2}} \delta(U)\left\{\left(\partial^{\prime} \partial+\partial^{\prime}\right)+\left[-\left(\left(\tau+\tau^{\prime *}\right)-\frac{\alpha|R|^{2}}{r}\left(\tau-\tau^{\prime *}\right)\right) \partial^{\prime}+\text { c.c. }\right]\right. \\
& \left.-2\left({ }^{\prime} \tau+\text { c.c. }\right)+2\left(\frac{\alpha|R|^{2}}{2 r}\right)^{2}\left|\tau-\tau^{\prime *}\right|^{2}\right\} f(\theta, \chi) \text {. }
\end{aligned}
$$

This completes the most arduous part of the calculation. It bears repeating that all quantities in Eq. (6.18) are understood to be evaluated at $r=r_{+}$, as mandated by the overall delta function. 


\subsection{Laplacian on the squashed sphere}

We could leave the result for $\square_{\perp} \hat{S}$ in the form of Eq. 6.18, but those familiar with the Dray-'t Hooft solution expect 2d Laplacians.

Our shift function $S$ and our horizon field $f$ have GHP weight $(-1,-1)$. In general, a weighted function $f_{h, h} \sim(h, h)$ has spin-weight $s \equiv h-\bar{h}=h-h=0$. The shockwave has $h=-1$, but without much fuss we can understand the situation for $s=0$ but arbitrary $h{ }^{31}$

By explicit computation on a function $f_{h, h}(\theta, \chi)$ of the Kruskal-like angular coordinates only, we find that the following combination of NP derivatives and GHP gauge fields reproduces the Laplacian on the squashed sphere.

$$
\delta^{\prime} \delta+\delta \delta^{\prime}-\left(\beta+\beta^{\prime *}\right) \delta^{\prime}-\left(\beta^{\prime}+\beta^{*}\right) \delta=\nabla_{2 \mathrm{~d}}^{2} .
$$

So unpacking the GHP derivatives according to their original definitions back in Eq. (2.29) provides the desired expression:

$$
\begin{aligned}
\left(ð^{\prime} ð+\partial^{\prime}\right) & f_{h, h}(\theta, \chi)=\left\{\nabla_{2 \mathrm{~d}}^{2}+\left[4 h\left(\beta-\beta^{\prime *}\right) \delta^{\prime}+c . c .\right]\right. \\
& \left.+2 h\left[\left(\delta^{\prime} \beta-\delta \beta^{\prime}+\text { c.c. }\right)+2\left(\left|\beta^{\prime}\right|^{2}-|\beta|^{2}\right)+4 h\left|\beta-\beta^{\prime *}\right|^{2}\right]\right\} f_{h, h}(\theta, \chi) .
\end{aligned}
$$

That is how our coveted $2 \mathrm{~d}$ spatial Laplacian manifests in our story. Its tragedy is that while we may find temporary solace in a familiar face, this yearning for camaraderie cost us the guidance of GHP covariance, without which we are hopelessly lost.

\section{$7 \quad$ Ricci tensor}

The trace-reversed Ricci tensor, being necessary to the gravitational field of a localized Source, the propensity of a massless particle to generate Curvature, shall now be realized.

\subsection{Relation to curvature scalars}

We emerge from the chrysalis of the tangent space by translating the usual prescription $R_{\mu \nu}=e_{\mu}^{a} e_{\nu}^{b} R_{a b}$ into the NP notation:

$$
\begin{aligned}
\frac{1}{2} R_{\mu \nu} & =l_{\mu}^{\prime} l_{\nu}^{\prime} \Phi_{00}+l_{\mu} l_{\nu} \Phi_{22}+\left[m_{\mu}^{\prime} m_{\nu}^{\prime} \Phi_{02}-\left(l_{\mu}^{\prime} m_{\nu}^{\prime}+m_{\mu}^{\prime} l_{\nu}^{\prime}\right) \Phi_{01}-\left(l_{\mu} m_{\nu}+m_{\mu} l_{\nu}\right) \Phi_{21}+c . c .\right] \\
& +\left(l_{\mu} l_{\nu}^{\prime}+l_{\mu}^{\prime} l_{\nu}+m_{\mu} m_{\nu}^{\prime}+m_{\mu}^{\prime} m_{\nu}\right) \Phi_{11}+\left(l_{\mu} l_{\nu}^{\prime}+l_{\mu}^{\prime} l_{\nu}-m_{\mu} m_{\nu}^{\prime}-m_{\mu}^{\prime} m_{\nu}\right) 3 \Pi .
\end{aligned}
$$

To evaluate the right-hand side, we first need to tilde everything (to calculate shifted quantities), and then we need to hat everything (to work in the horizon basis).

\footnotetext{
${ }^{31}$ Since complex conjugation exchanges $h$ and $\bar{h}$, only functions with $s=0$ can be taken real. We therefore assume $f_{h, h}^{*}=f_{h, h}$ for simplicity.

${ }^{32} \mathrm{~A}$ squashed sphere of radius $r$ has line element $d s^{2}=|R|^{2} d \theta^{2}+\frac{\left|R_{0}\right|^{4}}{|R|^{2}} \sin ^{2} \theta d \chi^{2} \equiv h_{i j} d x^{i} d x^{j}$, and the Laplacian derived from that is $\nabla_{2 \mathrm{~d}}^{2}=\frac{1}{|R|^{2}}\left[\partial_{\theta}^{2}+\left(\frac{\left|R_{0}\right|^{2}+a^{2} \sin ^{2} \theta}{|R|^{2}}\right) \cot \theta \partial_{\theta}+\frac{|R|^{4}}{\left|R_{0}\right|^{4}} \frac{1}{\sin ^{2} \theta} \partial_{\chi}^{2}\right]$. If $R_{i j}^{2 \mathrm{~d}}$ is the Ricci tensor derived from $h_{i j}$, then $\frac{1}{4} h^{i j} R_{i j}^{2 \mathrm{~d}}=\left.\operatorname{Re}(\mathcal{K})\right|_{r=r_{+}}$, the intrinsic curvature from Eq. 2.52 .
} 
We will specialize directly to the shockwave, so the only Ricci scalar that will shift is $\Phi_{22}$. Meanwhile, the unshifted geometry has only a nonzero $\Phi_{11}$. Therefore, we have for the full (i.e., including the unshifted part) Ricci tensor:

$$
\begin{aligned}
\frac{1}{2} \tilde{R}_{\mu \nu} & =\hat{\tilde{l}}_{\mu} \hat{\tilde{l}}_{\nu} \hat{\tilde{\Phi}}_{22}+\left(\hat{\tilde{l}} \mu \hat{\tilde{l}}_{\nu}^{\prime}+\hat{\tilde{l}}_{\mu}^{\prime} \hat{\tilde{l}}_{\nu}+\hat{\tilde{m}}_{\mu} \hat{\tilde{m}}_{\nu}^{\prime}+\hat{\tilde{m}}_{\mu}^{\prime} \hat{\tilde{m}}_{\nu}\right) \hat{\tilde{\Phi}}_{11} \\
& =\hat{l}_{\mu} \hat{l}_{\nu} \hat{\tilde{\Phi}}_{22}+\left(\hat{l}_{\mu} \hat{\tilde{l}}_{\nu}^{\prime}+\hat{\tilde{l}}_{\mu}^{\prime} \hat{l}_{\nu}+m_{\mu} m_{\nu}^{\prime}+m_{\mu}^{\prime} m_{\nu}\right) \Phi_{11} .
\end{aligned}
$$

In the second line we have removed the tildes for quantities that equal their unshifted counterparts, and we have removed the hats on quantities that do not get rescaled by factors of $U$ when passing from the standard frame to the horizon one.33

Recalling from Eq. (3.1) the premise that launched this travail in the first place, we isolate the part of the Ricci tensor that results from the shift:

$$
R_{\mu \nu}^{\mathrm{shift}}=2 \hat{l}_{\mu} \hat{l}_{\nu}\left(\hat{\tilde{\Phi}}_{22}+2 \hat{S} \Phi_{11}\right)
$$

Returning to our explicit expressions for the 1-forms in Eq. 2.47), we find:

$$
\left.\hat{l}\right|_{U=0}=\frac{\left|R_{+}\right|^{2}}{\alpha\left|R_{0+}\right|^{2}} d U .
$$

So we learn first of all that $R_{\mu \nu}^{\text {shift }}=R_{U U}^{\text {shift }} \delta_{\mu}^{U} \delta_{\nu}^{U}$, as promised.

\subsection{Relation to energy scalars}

Meanwhile, the energy tensor also admits an expansion analogous to Eq. (7.1):

$$
\begin{aligned}
4 \pi T_{\mu \nu} & =l_{\mu}^{\prime} l_{\nu}^{\prime} t_{00}+l_{\mu} l_{\nu} t_{22}+\left[m_{\mu}^{\prime} m_{\nu}^{\prime} t_{02}-\left(l_{\mu}^{\prime} m_{\nu}^{\prime}+m_{\mu}^{\prime} l_{\nu}^{\prime}\right) t_{01}-\left(l_{\mu} m_{\nu}+m_{\mu} l_{\nu}\right) t_{21}+c . c .\right] \\
& +\left(l_{\mu} l_{\nu}^{\prime}+l_{\mu}^{\prime} l_{\nu}+m_{\mu} m_{\nu}^{\prime}+m_{\mu}^{\prime} m_{\nu}\right) t_{11}+\left(l_{\mu} l_{\nu}^{\prime}+l_{\mu}^{\prime} l_{\nu}-m_{\mu} m_{\nu}^{\prime}-m_{\mu}^{\prime} m_{\nu}\right) 3 t_{\Pi}
\end{aligned}
$$

Anticipating the required energy tensor term by term, we conclude:

$$
8 \pi T_{\mu \nu}^{\text {shift }}=2 \hat{l}_{\mu} \hat{l}_{\nu}\left(\hat{\tilde{t}}_{22}+2 \hat{S} t_{11}\right) .
$$

Given that the background Einstein equation is, by construction, $\Phi_{11}=t_{11}$ [recall Eq. (2.71]], all we need is a $t_{22}$ such that

$$
\hat{\tilde{\Phi}}_{22}=\hat{\tilde{t}}_{22} .
$$

The whole point of this tale is that the correction to the left-hand side can be interpreted as the backreaction from a massless particle on the future horizon, so that is what will populate the right-hand side. In this paper we focus on the geometry instead of the field theory, so let us leave that aside and press on.

\footnotetext{
${ }^{33}$ There is no need to place a hat on the Ricci tensor, because by construction it is invariant under GHP transformations of the frame.
} 


\subsection{Final result for $\Phi_{22}$}

Returning to our earlier calculation of $\partial \hat{S}$ [Eq. (6.9)], multiplying by $\tau^{*}-\tau^{\prime}$, integrating by parts, and adding the complex conjugate, we obtain the remaining first-derivative terms:

$$
\left(\tau-\tau^{* *}\right) ð^{\prime} \hat{S}+\text { c.c. }=-\frac{c}{2|R|^{2}} \delta(U)\left\{\left(\tau-\tau^{\prime *}\right) ð^{\prime}+\left(\tau^{*}-\tau^{\prime}\right) \partial+\frac{\alpha|R|^{2}}{r}\left|\tau-\tau^{\prime *}\right|^{2}\right\} f(\theta, \chi) \text {. }
$$

Next take the general shifted $\Phi_{22}$ from Eq. (5.7), hat it, and recognize that $\hat{\rho}^{\prime} \hat{\mathrm{p}} \hat{S}$ and $\left(\hat{\rho}-\hat{\rho}^{*}\right)\left(\hat{\rho}^{\prime}-\hat{\rho}^{*}\right)$ go to zero at $U=0$.

But $\hat{\rho} \hat{\mathrm{p}}^{\prime} \hat{S}$ is more subtle, since within $\hat{D}^{\prime}$ lurks $\partial_{U}$. Applying Eq. 6.7), we obtain

$$
\operatorname{Re}\left(\hat{\rho} \hat{D}^{\prime} \hat{S}\right)=\alpha r_{+} \frac{\left|R_{0+}\right|^{2}}{\left|R_{+}\right|^{4}} \hat{S}=-\left.\frac{1}{2}\left[ð \tau+\chi^{\prime} \tau^{*}+2|\tau|^{2}+2 \operatorname{Re}\left(\Psi_{2}\right)\right]\right|_{r=r_{+}} \hat{S} .
$$

Because $\left.\hat{\rho}\right|_{U=0}=0$, the terms involving $\hat{\varepsilon}^{\prime}$ and $\hat{\varepsilon}^{*}$ drop out, leaving us with $\operatorname{Re}\left(\hat{\rho} \hat{\mathrm{p}}^{\prime} \hat{S}\right)=$ $\operatorname{Re}\left(\hat{\rho} \hat{D}^{\prime} \hat{S}\right)=-\operatorname{Re}\left(\hat{\mathrm{p}}^{\prime} \hat{\rho}\right) \hat{S}$.

Putting all this together (and using $\Phi_{00}=\Pi=0$ ), we reduce our shifted $\Phi_{22}$ to the relatively compact form:

$$
\hat{\tilde{\Phi}}_{22}=\frac{1}{2}\left\{\square_{\perp}+\left(\tau-\tau^{\prime *}\right) ð^{\prime}+\left(\tau^{*}-\tau^{\prime}\right) ð+\left(ð^{\prime} \tau+\text { c.c. }\right)+2|\tau|^{2}+2 \operatorname{Re}\left(\Psi_{2}\right)\right\} \hat{S} .
$$

Enlisting our result for $\square_{\perp} \hat{S}$ in Eq. (6.18) and the relation $4|\tau|^{2}=\left|\tau+\tau^{* *}\right|^{2}+\left|\tau-\tau^{\prime *}\right|^{2}$, we finally obtain the beautiful, exquisite, magical expression

$$
\hat{\tilde{\Phi}}_{22}=-\frac{c}{4|R|^{2}} \delta(U) \mathscr{D} f(\theta, \chi)
$$

where the differential operator $\mathscr{D}$ is

$$
\begin{aligned}
\mathscr{D} & =\chi^{\prime} \partial+\partial^{\prime}+\left[-\left(\tau+\tau^{\prime *}\right)+\left(1+\frac{\alpha|R|^{2}}{r}\right)\left(\tau-\tau^{\prime *}\right)\right] \partial^{\prime}+\left[-\left(\tau^{*}+\tau^{\prime}\right)+\left(1+\frac{\alpha|R|^{2}}{r}\right)\left(\tau^{*}-\tau^{\prime}\right)\right] \partial \\
& +2 \operatorname{Re}\left(\Psi_{2}\right)-\left(\chi^{\prime} \tau+\partial \tau^{*}\right)+\frac{1}{2}\left|\tau+\tau^{\prime *}\right|^{2}+\frac{1}{2}\left(1+\frac{\alpha|R|^{2}}{r}\right)^{2}\left|\tau-\tau^{\prime *}\right|^{2} .
\end{aligned}
$$

This is our final result.

It is expressed in terms of quantities that have innate geometrical significance, in that each operator has a definite GHP weight. When $a=0$, we obtain 34

$$
\left.\mathscr{D}\right|_{a=0}=ð^{\prime} \partial+\partial \nearrow^{\prime}+2 \operatorname{Re}\left(\Psi_{2}\right) \text {. }
$$

As could be anticipated from the Type $\mathrm{D}$ character of the background, we see that it is part of the Weyl tensor, $\operatorname{Re}\left(\Psi_{2}\right)$, not the intrinsic curvature, $\operatorname{Re}(\mathcal{K})$, that appears most naturally

\footnotetext{
${ }^{34}$ At $r=r_{+}$, we have $\left.\operatorname{Re}\left(\Psi_{2}\right)\right|_{a=0}=-\frac{\alpha}{r_{+}}$.
} 
in the GHP-covariant form of the shifted $\Phi_{22}$ for generic values of the angular momentum.

On the other hand, the intrinsic curvature presents itself when we trade the GHP-covariant derivatives for the $2 \mathrm{~d}$ Laplacian plus its associated ejecta. We first expand $\partial f=[\delta+2(-1) \beta-$ $\left.2(-1) \beta^{\prime *}\right] f$ and specialize Eq. (6.20) to $h=-1$. Then we shuffle the terms around using numerical relations like 35

$$
\beta^{\prime}-\beta^{*}=\tau^{\prime} \quad(\text { Kerr-Newman })
$$

and

$$
\left|\beta^{\prime}\right|^{2}-|\beta|^{2}=\frac{a^{2}}{2|R|^{4}} \quad(\text { Kerr-Newman }) .
$$

In this way we obtain the following alternative form for Eq. (7.12):

$$
\begin{aligned}
\mathscr{D} & =\nabla_{2 \mathrm{~d}}^{2}+\frac{1-\alpha R}{r}\left(\tau-\tau^{\prime *}\right) R^{*} \delta^{\prime}+\frac{1-\alpha R^{*}}{r}\left(\tau^{*}-\tau^{\prime}\right) R \delta \\
& +2 r \alpha\left\{-2\left(\frac{|R|^{2}}{\left|R_{0}\right|^{2}} \operatorname{Re}(\mathcal{K})+\frac{2 a^{2}}{|R|^{4}}\right)+\left|\tau+\tau^{*}\right|^{2}+\left[1+r \alpha\left(\frac{|R|^{2}}{2 r^{2}}\right)^{2}\right]\left|\tau-\tau^{\prime *}\right|^{2}\right\} .
\end{aligned}
$$

We will refer to the coefficient of $f(\theta, \chi)$ in $\hat{\tilde{\Phi}}_{22}$, encapsulated by the term in Eq. 7.16 without any derivatives, as the "mass term." It is organized in terms of the intrinsic curvature at the horizon [recall Eq. (2.52)] and quantities proportional to some power of the angular momentum. Expressed in this way, the mass term reeks of Kaluza-Klein, but we will leave that for another day. Regardless, this form shows clearly which terms go to zero as we turn off the rotation.

When $a=0$ (but $Q \neq 0$ ), we recover the known spherically symmetric answer: ${ }^{36}$

$$
\left.\hat{\tilde{\Phi}}_{22}\right|_{a=0}=-\frac{c}{4 r_{+}^{2}} \delta(U)\left(\nabla_{2 \mathrm{~d}}^{2}-\frac{2 \alpha}{r_{+}}\right) f(\theta, \varphi) .
$$

While the geometrical significance of the mass term in Eq. (7.16) eludes us, the physical significance of the overall factor of $\alpha$ in shockwave geometries has been emphasized by others ${ }^{37}$ In the extremal limit, which in this case is $a^{2}+Q^{2}=M^{2}$ and hence $r_{-}=r_{+}$, the surface gravity $\alpha$ goes to zero (as usual), and the entire mass term vanishes.

As far as we know, the first to point this out in the spherically symmetric situation was Sfetsos, who interpreted it as a breakdown of the solution [23]. The effect was recently revisited by Leichenauer in the context of entanglement between the conformal field theories dual to the asymptotically-AdS generalization of the Reissner-Nordström black hole [48]. And in the context of scattering, the vanishing of the mass term in the operator $\mathscr{D}$ is what Maldacena and Stanford call the " $\beta J$ enhancement" of the amplitude [16].

\footnotetext{
${ }^{35}$ It is possible that these relations embody some hidden meaning. But the two sides of Eq. (7.14) do not transform in the same way under Eq. 2.17), so we hesitate to dig deeper.

${ }^{36}$ At $r=r_{+}$, we have $\left.\operatorname{Re}(\mathcal{K})\right|_{a=0}=\frac{1}{2 r_{+}^{2}}$. Also, when $a=0$ the delayed angle $\chi$ becomes the ordinary azimuthal angle $\varphi$.

${ }^{37}$ We thank Douglas Stanford for explaining this to us.
} 
But let us not get ahead of ourselves. In this paper we are concerned exclusively with the single-shockwave geometry and its interpretation within general relativity. The sun will rise tomorrow, and we will have another opportunity to traverse that wormhole.

\section{Discussion}

Inspired by 't Hooft's S-matrix approach to quantum gravity and Kitaev's recent revival thereof, we have generalized the Dray-'t Hooft gravitational shockwave to the Kerr-Newman black hole using the method of spin coefficients.

We have not solved the resulting Green's function equation, $\mathscr{D} f \propto \delta^{2}\left(\vec{x}_{\perp}\right)$. Since $\mathscr{D}$ is analytic near $a=0$, we could perturb around the Dray-'t Hooft integral formula [19]. Or maybe we should expand in spheroidal harmonics, but we would probably have to resort to numerics for anything beyond a rudimentary understanding ${ }^{38}$ On a different tack, we could perturb other backgrounds by shifting the frame: Shockwaves on Kerr-AdS might eventually lead to precise statements about chaos in a putative dual field theory ${ }^{39}$

We will conclude with a pedantic remark about the effective action for the horizon field. Given a classical equation of motion, we should ask what variational principle could lead to it. Since the Ricci tensor is linear in $f(\theta, \chi)$, our equation of motion is linear in the field, so we might expect a quadratic action.

But the Lagrangian is proportional to the Einstein-Hilbert curvature $\Pi$, which we have already seen is linear in $f$. What to make of this? Recall that if the "equation of motion" is actually a constraint - which in this case it is - then it should be implemented in the calculus of variations by introducing a Lagrange multiplier.

Consider a path integral over all classical fields $f(\theta, \chi)$ that satisfy $\mathscr{D} f=0.40$

$$
\mathcal{Z} \equiv \int \mathcal{D} f \delta(\mathscr{D} f)=\int \mathcal{D} f \mathcal{D} f^{\prime} e^{i \int d^{2} x f^{\prime} \mathscr{D} f}
$$

We have used the Fourier representation of the delta function and thereby concocted a classical field $f^{\prime}$, which serves as a Lagrange multiplier for the equation $\mathscr{D} f=0$.

The argument of the exponential in Eq. (8.1) is 't Hooft's effective action [9]. This straightforward interpretation of the constraint for the horizon field provides a path-integral sense in which the two shockwaves are canonically conjugate variables.

\footnotetext{
${ }^{38}$ Dray and 't Hooft themselves "have not attempted to perform the integration explicitly" for their result [19]. Sfetsos, for his part, did elaborate somewhat on his solutions in Appendix D of his paper [23].

${ }^{39}$ We thank Nick Hunter-Jones for encouragement in this direction.

${ }^{40}$ For the sake of brevity we are only considering the gravitational part of the action. More generally there should be an $f$-independent function on the right-hand side of the constraint.
} 


\section{Acknowledgments}

We thank Jan Willem Dalhuisen, Aaron Zimmerman, David Nichols, Christopher White, Dave Aasen, Nick Hunter-Jones, Alex Rasmussen, Yonah Lemonik, Douglas Stanford, and Saul Teukolsky for insightful discussions at various points in this endeavor. Y. B. especially thanks Justin Wilson, Leo Stein, and Alexei Kitaev. J. S. especially thanks Dirk Bouwmeester. Y. B. is funded by the Institute for Quantum Information and Matter (NSF Grant PHY-1125565) with support from the Simons Foundation (award number 376205).

\section{A Signature change}

In this appendix we sail from West to East, scrupulously marking all signs in our wake. Relics will be tagged by overbars.

\section{A.1 Basic assumptions}

We begin by flipping the signs of both the base space and tangent space metrics:

$$
g_{\mu \nu} \equiv \zeta \bar{g}_{\mu \nu}, \eta_{a b} \equiv \zeta \bar{\eta}_{a b}, \quad \zeta \equiv-1 \text {. }
$$

In terms of the corresponding frames, we have $g_{\mu \nu}=\eta_{a b} e_{\mu}^{a} e_{\nu}^{b}, \bar{g}_{\mu \nu}=\bar{\eta}_{a b} \bar{e}_{\mu}^{a} \bar{e}_{\nu}^{b}, \eta_{a b}=g_{\mu \nu} e_{a}^{\mu} e_{b}^{\nu}$, and $\bar{\eta}_{a b}=\bar{g}_{\mu \nu} \bar{e}_{a}^{\mu} \bar{e}_{b}^{\nu}$. Defining $e_{a \mu} \equiv \eta_{a b} e_{\mu}^{b}$ and $\bar{e}_{a \mu} \equiv \bar{\eta}_{a b} \bar{e}_{\mu}^{b}$, we obtain from Eq. (A.1):

$$
e_{\mu}^{a} e_{a \nu}=\zeta \bar{e}_{\mu}^{a} \bar{e}_{a \nu}, \quad e_{a}^{\mu} e_{b \mu}=\zeta \bar{e}_{a}^{\mu} \bar{e}_{b \mu} .
$$

Relative to Chandrasekhar [31], our null vectors $\left(l^{\mu}, l^{\prime \mu}, m^{\mu}, m^{\prime \mu}\right)$ will not flip sign, in which case our null forms $\left(l_{\mu}, l_{\mu}^{\prime}, m_{\mu}, m_{\mu}^{\prime}\right) \equiv\left(g_{\mu \nu} l^{\nu}, g_{\mu \nu} l^{\prime \nu}, g_{\mu \nu} m^{\nu}, g_{\mu \nu} m^{\prime \nu}\right)$ will. This is a choice.

From this - with attention to the fact that the basis is null-we infer:

$$
e_{a}^{\mu} \equiv \bar{e}_{a}^{\mu}, \quad e_{\mu}^{a} \equiv \bar{e}_{\mu}^{a} .
$$

Neither $e_{a}^{\mu}$ nor $e_{\mu}^{a}$ flips sign. What does flip sign is the quantity with both indices lowered:

$$
e_{a \mu}=\zeta \bar{e}_{a \mu} .
$$

\section{A.2 Spin coefficients flip sign}

If we insert the above definitions into $d e^{a}+\omega^{a}{ }_{b} \wedge e^{b}=0$, we will find that the spin connection with one index up and one index down does not flip sign:

$$
\omega^{a}{ }_{b}=\bar{\omega}_{b}^{a} .
$$

So $\omega_{a b}$ does flip sign. Unpacking the 1-form index and recognizing that $d x^{\mu}=d \bar{x}^{\mu}$, we find $\left(\omega_{\mu}\right)_{a b}=\zeta\left(\bar{\omega}_{\mu}\right)_{a b}$. Recalling Eq. 2.12), we conclude that the spin coefficients flip sign:

$$
\gamma_{a b c}=\zeta \bar{\gamma}_{a b c} .
$$

Meanwhile, because of Eq. A.4, the null Cartan equations are the same in either signature. So Eq. 2.32 looks exactly the same as Eq. (4.13.44) in Spinors and Spacetime [32]. 


\section{A.3 Curvature scalars do not flip sign}

Next up, curvature. Since $\omega_{b}^{a}$ does not flip sign, neither does $\Omega_{b}^{a} \equiv d \omega_{b}^{a}+\omega_{c}^{a} \wedge \omega_{b}^{c}$ :

$$
\Omega_{b}^{a}=\bar{\Omega}_{b}^{a} .
$$

So $\Omega_{a b}$ does flip sign. As an unavoidable consequence, the Riemann tensor in the tangent space with all indices down, $R_{a b c d} \equiv\left(\Omega_{\mu \nu}\right)_{a b} e_{c}^{\mu} e_{d}^{\nu}$, flips sign:

$$
R_{a b c d}=\zeta \bar{R}_{a b c d} \text {. }
$$

It is misleading to simply assert that the Newman-Penrose equations remain fixed upon changing the metric signature, as if it were to follow as night the day.

Crucially, the Weyl scalars are defined from $C_{a b c d}$, which in turn is defined from $R_{a b c d}$ [recall Eq. [2.62] - this quantity flips sign under a change of signature:

$$
C_{a b c d}=\zeta \bar{C}_{a b c d} .
$$

Should we fashion an extra sign in the definition of the Weyl scalars to obviate this? No. Beside the sign from Eq. A.8, there is also an overall sign choice in the definition of the curvature scalars - by sheer happenstance, our conventions in Eq. (2.63) automatically cancel this additional sign compared to the GHP equations as traditionally written [34].

Meanwhile, since $R_{b c d}^{a}=\eta^{a e} R_{e b c d}$ and $R_{a b c d}=\zeta \bar{R}_{a b c d}$, the Ricci tensor in the tangent space does not flip sign:

$$
R_{a b} \equiv R_{a c b}^{c}=\bar{R}_{a c b}^{c} \equiv \bar{R}_{a b} .
$$

For the Ricci scalars in Eq. 2.56 we do commission a sign relative to the standard references.

The Einstein-Hilbert curvature sprouts yet another sign:

$$
\eta^{a b} R_{a b}=\zeta \bar{\eta}^{a b} \bar{R}_{a b}
$$

To maintain the sanctity of the GHP equations, we must begrudgingly define

$$
\Pi \equiv-\frac{1}{24} \eta^{a b} R_{a b}=-\zeta \frac{1}{24} \bar{\eta}^{a b} \bar{R}_{a b}=+\bar{\Pi}
$$

\section{A.4 Extra sign in GHP derivatives}

Before docking we must ensure that the Icelandic runes make sense. Consider the GHP derivatives as defined by Penrose and Rindler [32]:

$$
\begin{aligned}
& \overline{\mathrm{p}} \equiv \bar{D}-2 h \bar{\varepsilon}-2 \bar{h} \bar{\varepsilon}^{*}, \quad \bar{\delta} \equiv \bar{\delta}-2 h \bar{\beta}+2 \bar{h} \bar{\beta}^{\prime *}, \\
& \overline{\mathrm{p}}^{\prime} \equiv \bar{D}^{\prime}+2 h \bar{\varepsilon}^{\prime}+2 \bar{h} \bar{\varepsilon}^{*}, \quad \bar{\delta}^{\prime} \equiv \bar{\delta}^{\prime}+2 h \bar{\beta}^{\prime}-2 \bar{h} \bar{\beta}^{*} .
\end{aligned}
$$

Explicitly verifying their GHP covariance on a weighted test function, we see that a certain crucial sign emerges as a result of whether $l^{\mu} l_{\mu}^{\prime}=-m^{\mu} m_{\mu}^{\prime}$ is +1 or -1 . It is this sign that determines the extra signs in Eq. A.13) relative to those in Eq. (2.29). 


\section{References}

[1] D. Christodoulou. Reversible and irreversible transformations in black-hole physics. Phys. Rev. Lett., 25(22):1596-1597, 1970.

[2] R. Penrose and R. M. Floyd. Extraction of rotational energy from a black hole. Nat. Phys. Sci., 229:177-179, 1971.

[3] B. Carter. Rigidity of a black hole. Nat. Phys. Sci., 238:71-72, 1972.

[4] J. D. Bekenstein. Black holes and entropy. Phys. Rev. D, 7(8):2333-2346, 1973.

[5] J. M. Bardeen, B. Carter, and S. W. Hawking. The four laws of black hole mechanics. Commun. Math. Phys., 31:161-170, 1973.

[6] S. Hawking. Black hole explosions? Nature, 248:30-31, 1974.

[7] S. Hawking. Particle creation by black holes. Commun. Math. Phys., 43:199-220, 1975.

[8] A. Strominger and C. Vafa. Microscopic origin of the Bekenstein-Hawking entropy. Phys. Lett. B, 379:99-104, 1996.

[9] G. 't Hooft. The black hole interpretation of string theory. Nucl. Phys. B, (1):138-154, 1990 .

[10] G. 't Hooft. The scattering matrix approach for the quantum black hole, an overview. Int. J. Mod. Phys. A11, pages 4623-4688, 1996.

[11] S. H. Shenker and D. Stanford. Black holes and the butterfly effect. JHEP03, 67, 2014.

[12] S. H. Shenker and D. Stanford. Stringy effects in scrambling. JHEP05, 132, 2015.

[13] A. Kitaev. A simple model of quantum holography. Talk given at the Kavli Institute for Theoretical Physics at the University of California, Santa Barbara (Feb. 12, 2015; Apr. 7, 2015; May 27, 2015).

[14] J. Maldacena, D. Stanford, and Z. Yang. Conformal symmetry and its breaking in two-dimensional nearly anti-de Sitter space. Prog. Theor. Exp. Phys., 2016(12):12C104, 2016.

[15] A. Kitaev and S. J. Suh. The soft mode in the Sachdev-Ye-Kitaev model and its gravity dual. JHEP05, 183, 2018.

[16] J. Maldacena and D. Stanford. Remarks on the Sachdev-Ye-Kitaev model. Phys. Rev. D, 94:106002, 2016.

[17] A. Strominger. $A d S_{2}$ quantum gravity and string theory. JHEP01, 8, 1999.

[18] E. Witten. An SYK-like model without disorder. arXiv:1610.09758 [hep-th]. 
[19] T. Dray and G. 't Hooft. The gravitational shock wave of a massless particle. Nucl. Phys. B, 253:173-188, 1985.

[20] P. C. Aichelburg and R. U. Sexl. On the gravitational field of a massless particle. General Relativity and Gravitation, 2(4):303-312, 1971.

[21] R. Penrose. In L. O'Raifeartaigh, editor, General Relativity: papers in honour of J. L. Synge. Clarendon, Oxford, 1972.

[22] R. Alonso and N. Zamorano. Generalized Kerr-Schild metric for a massless particle on the Reissner-Nordström horizon. Phys. Rev. D, 35(6):1798-1801, 1987.

[23] K. Sfetsos. On gravitational shock waves in curved spacetimes. Nucl. Phys. B, 436:721745, 1995.

[24] Y. Kiem, H. Verlinde, and E. Verlinde. Black hole horizons and complementarity. Phys. Rev. D, 52(12):7053-7065, 1995.

[25] J. Polchinski. Chaos in the black hole S-matrix. arXiv:1505.08108 [hep-th].

[26] A. Almheiri, D. Marolf, J. Polchinski, and J. Sully. Black holes: complementarity or firewalls? JHEP, 2:062, 2013.

[27] D. Marolf and J. Polchinski. Gauge-gravity duality and the black hole interior. Phys. Rev. Lett., 111:171301, 2013.

[28] B. P. Abbott al. Observation of gravitational waves from a binary black hole merger. Phys. Rev. Lett., 116(061102), 2016.

[29] H. Balasin. Generalized Kerr-Schild metrics and the gravitational field of a massless particle on the horizon. Class Quantum Grav., 17:1913-1920, 2000.

[30] A. H. Taub. Generalized Kerr-Schild space-times. Annals of Physics, 134:326-372, 1981.

[31] S. Chandrasekhar. The Mathematical Theory of Black Holes. Oxford University Press, April 281983.

[32] R. Penrose and W. Rindler. Spinors and space-time. Cambridge University Press, 1987.

[33] E. Newman and R. Penrose. An approach to gravitational radiation by a method of spin coefficients. J. Math. Phys., 3(566):566-578, 1962.

[34] R. Geroch, A. Held, and R. Penrose. A space-time calculus based on pairs of null directions. J. Math. Phys., 14(874):874-881, 1973.

[35] R. H. Boyer and R. W. Lindquist. Maximal analytic extension of the Kerr metric. J. Math. Phys., 8:265-281, 1967.

[36] E. T. Newman and A. I. Janis. Note on the Kerr spinning-particle metric. J. Math. Phys., 6:915-917, 1965. 
[37] R. K. Sachs. Gravitational waves in general relativity. VI. The outgoing radiation condition. In Proc. Roy. Soc. 264, No. 1318, 1961.

[38] P. Szekeres. On the propagation of gravitational fields in matter. J. Math. Phys, 7:751761, 1966.

[39] R. K. Sachs. Gravitational radiation. In Relativity Groups and Topology. Lectures Delivered at Les Houches During the 1963 Session of the Summer School of Theoretical Physics, pages 521-562. DeWitt and DeWitt, published by Gordon \& Breach, Science Publishers, Inc, 1964.

[40] P. A. M. Dirac. Forms of relativistic dynamics. Rev. Mod. Phys., 21(3):392-399, 1949.

[41] L. Smarr. Surface geometry of charged rotating black holes. Phys. Rev. D, 7(2):289-295, 1973.

[42] P. Szekeres. The gravitational compass. J. Math. Phys., 6(9):1387-1391, 1965.

[43] A. Z. Petrov. The classification of spaces defining gravitational fields. General Relativity and Gravitation, 32(8):1665-1685, 2000. [This is an updated version of: A. Z. Petrov. On spaces defining gravitational fields. Dokl. Akad. Nauk SSSR, XXXI (1951) 149-152].

[44] J. B. Griffiths. Colliding Plane Waves in General Relativity. Clarendon Press, Oxford, (1991); Dover reprint 2016.

[45] M. Fels and A. Held. Kerr-Schild rides again. General Relativity and Gravitation, pages 61-68, 1989.

[46] R. A. Matzner. Behavior of ray optics in the Dray-'t Hooft geometry. Nucl. Phys. B, 266:661-668, 1986.

[47] B. O'Neill. The Geometry of Kerr Black Holes. A K Peters, Ltd., Wellesley, Massachusetts, 1995.

[48] S. Leichenauer. Disrupting entanglement of black holes. Phys. Rev. D, 90(046009), 2014. 